\title{
GIANTS IN OLD OPEN CLUSTERS: TEMPERATURES, LUMINOSITIES, AND ABUNDANCES FROM INFRARED PHOTOMETRY
}

\author{
Mark L. Houdashelt And Jay A. Frogel ${ }^{1,2}$ \\ Department of Astronomy, The Ohio State University, 174 West 18th Avenue, Columbus, Ohio 43210 \\ JUDITH G. COHEN ${ }^{2}$ \\ Palomar Observatory, California Institute of Technology, Pasadena, California 91125 \\ Received 21 June 1991; revised 29 August 1991
}

\begin{abstract}
We present infrared observations for more than 100 red giants and clump stars in eight old open clusters. We have assembled the best available optical photometry for these stars and determined a consistent set of integrated physical parameters (reddening, distance modulus, abundance, and age) for the clusters. From color-color and color-magnitude diagrams that make use of both the infrared and optical data, we are able to identify quite a few stars as probable field stars rather than cluster members. However, because of a general scarcity of bright stars, it is often difficult to distinguish between cluster members on the asymptotic giant branch and field interlopers. In a $(U-V)_{0},(V-K)_{0}$ plot, stars from the most metal-poor open clusters tend to lie between the relations defined by field and globular cluster giants. On the other hand, nearly all of the open cluster stars lie near the field giant line in a $(J-H)_{0}$, $(H-K)_{0}$ plot. The mean CO strengths of the giants in each open cluster show a range consistent with the optically determined range in $[\mathrm{Fe} / \mathrm{H}]$, but the correlation between these two quantities is weak, probably because of the small total range of each and the significant uncertainties in $[\mathrm{Fe} / \mathrm{H}]$. The results for the open clusters, though, are consistent with the relation between $\mathrm{CO}$ and $[\mathrm{Fe} / \mathrm{H}]$ established for globular clusters and considerably strengthen that relation near the solar metallicity end. For these eight open clusters, there is a modest linear correlation between $[\mathrm{Fe} / \mathrm{H}]$ and age which shows a gradient in metallicity of about -0.1 dex per Gyr and gives $[\mathrm{Fe} / \mathrm{H}] \sim-0.6$ for $\tau=5 \mathrm{Gyr}$, the age of the sun. The relation is quite similar in slope and zero point to that exhibited by clusters in the Large Magellanic Cloud. If the open cluster data are adjusted for the galactic radial metallicity gradient, the age-metallicity relation becomes intermediate to those of the Large Magellanic Cloud and the solar neighborhood. This could be an indication that the old open clusters are representative of the stellar population of the galactic thick disk. The temperatures of the cluster giant branches determined from infrared observations are tightly correlated with the optically derived values for age and $[\mathrm{Fe} / \mathrm{H}]$. Also, there is general agreement between the location of the cluster giant branches in an $H-R$ diagram determined from infrared photometry and the predictions of the Revised Yale Isochrones. Specific differences that exist between the theoretical and semiempirical parameters can at least partially be attributed to uncertainties in the (primarily) optical data and/or the presence of convective overshooting.
\end{abstract}

\section{INTRODUCTION}

This paper presents and analyzes infrared data for the brightest giant stars in eight old open clusters - Melotte 66, M67 (NGC 2682), NGC 2204, NGC 2243, NGC 2420, NGC 2477, NGC 2506, and NGC 2660. Similar data have been used to derive bolometric luminosities, effective temperatures, and metallicities for giants in globular clusters (Cohen et al. 1978, hereafter referred to as CFP; Frogel et al. 1981, hereafter referred to as FPC81; Frogel et al. 1983, hereafter referred to as FCP; Frogel et al. 1983, hereafter referred to as FPC83). These empirically determined parameters have provided valuable tests of stellar evolutionary theory and a new approach to abundance determinations.

The approach we take in analyzing the data for the open cluster giants is similar to that taken for the globular clusters in the references cited above. Section 2 discusses the open cluster parameters obtained from the literature: optical pho-

' On the staff of Cerro Tololo Inter-American Observatory, National Optical Astronomy Observatories, when these observations were made.

${ }^{2}$ Guest Observer, Las Campanas Observatory, Carnegie Institution of Washington. tometry, reddening, distance modulus, age, metallicity, and membership probability. We describe the infrared observing program in Sec. 3. Sections 4 and 5 present the color-magnitude diagrams (CMDs) and color-color plots, respectively; stars which may be field stars or otherwise peculiar are noted here. Section 4 also provides an extensive review of the literature pertaining to the bright giants in these clusters. Thus, readers primarily interested in our analysis and results may wish to simply examine the cluster CMDs given in Figs. 1-8 and skip directly to Sec. 5 of the text.

Individual stellar bolometric magnitudes and effective temperatures are derived in Sec. 6; giant branch (GB) parameters analogous to those developed for the globular clusters are also presented. However, we are unable to examine the open cluster GBs at the same luminosities used for the globular clusters $\left(M_{K}=-5.5\right.$ and $\left.M_{\text {bol }}=-3.0\right)$ because we observed giants this bright in only two of the open clusters. Unfortunately, the sparseness of the GBs also prevents us from making a meaningful comparison with results obtained from a study of clusters of similar age in the Magellanic Clouds (Frogel et al. 1990) and with predictions of asymptotic giant branch (AGB) evolution.

The age-metallicity relation for the eight open clusters is 
discussed in Sec. 7. In Sec. 8, the data are compared with the Revised Yale Isochrones (Green et al. 1987) to examine the compatibility of the observed GB parameters with those predicted. Possible revisions of these isochrones and the cluster parameters chosen from the literature are discussed. Section 9 is a summary of our results.

\section{CLUSTER PARAMETERS FROM THE LITERATURE}

The clusters observed in our program are listed in Table 1. This table provides the values of the reddening, distance modulus, age, and metallicity we use for each cluster; galactic coordinates and galactocentric distances (assuming $R_{\odot}$ $=8.5 \mathrm{kpc}$ ) are also included in Table 1 . Published $U B V$ photometry for every cluster star we observed in the infrared is given in Tables 2-9. If more than one source is noted, a straight average of the literature values has been taken. We estimate typical uncertainties of $\pm 0.05 \mathrm{mag}$ in the optical data and uncertainties no larger than $\pm 0.05 \mathrm{mag}$ in $\mathrm{E}(B-V), \pm 0.50 \mathrm{mag}$ in $(m-M)_{0}, \pm 1.0 \mathrm{Gyr}$ in cluster age, and $\pm 0.3 \mathrm{dex}$ in $[\mathrm{Fe} / \mathrm{H}]$. The following subsections explain the considerations that have gone into the selection of the photometry and the physical parameters from the literature.

\subsection{UBVR Photometry}

Photoelectric and CCD UBVR magnitudes and colors were preferred over photographic values and have been used exclusively whenever possible. Photographic photometry was adopted only when no photoelectric data were found but was always consulted when assessing the variability of cluster members. Because $(V-R)_{0}$ colors simply serve to enhance the determination of the cluster fiducial parameters (see Sec. 6), they are not given in Tables 2-9; however, these colors were only available for portions of two clusters. For M67, the $V-R$ photometry presented by Taylor \& Joner (1988) was preferred; Schild (1983, 1985), Janes \& Smith (1984), Taylor \& Joner (1985), and Joner \& Taylor (1988) were secondary sources. For NGC 2204, Dawson's (1981) values of $V-R$ were used.

\subsection{Reddenings}

In general, any "recent" photometric reddening determination, based on either cluster members or nearby field stars, was used to derive an average cluster reddening. However, when a reference cited more than one possible reddening for a cluster, a choice of the most consistent value was required. For Melotte 66, the reddening value of Hawarden (1976b) which includes stars 2261 and 2239 was selected; also, $\mathrm{E}(B-V)$ taken from Hawarden (1975) for NGC 2420 includes star $\mathbf{F}$.

The literature averages of $\mathrm{E}(B-V)$ have not been adopted for M67, NGC 2477, and NGC 2660. Because of the low galactic latitudes of the latter two clusters, field stars are suspected to contribute to their apparently variable reddenings. For this reason, the reddening determination of Hartwick \& Hesser (1973) derived from observations of nearby field stars alone was considered to be most appropriate for the giants in NGC 2660. Individual reddenings were derived for the NGC 2477 stars because this cluster shows a much more nonuniform extinction pattern. The $\mathrm{E}(B-V)$ values we use for the NGC 2477 giants are given in Table 7; their use greatly reduces the GB scatter seen in the cluster's CMD (Fig. 6) when either a single reddening is assumed for the entire cluster or the individual stellar reddenings of Hartwick et al. (1972, hereafter referred to as HHM) are used. Justification of the specific reddening values chosen for NGC 2477 and M67 is presented in Sec. 5.1.

\subsection{Distance Moduli and Ages}

Published distance moduli and ages were chosen only from isochrone fits to CMDs in which the isochrones of Ciardullo \& Demarque (1977), those of VandenBerg (1985) or the Revised Yale Isochrones were utilized. Because of the uncertainties in the derived values of $(m-M)_{0}$

TABLE 1. Cluster parameters.

\begin{tabular}{|c|c|c|c|c|c|c|c|c|c|c|c|}
\hline Cluster & $E(B-V)$ & Sources & $(m-M)_{0}$ & Sources & Age $^{a}$ & Sources & {$[\mathrm{Fe} / \mathrm{H}]$} & Sources & $l^{\mathrm{b}}$ & $b^{b}$ & $\mathrm{R}^{\mathrm{c}}$ \\
\hline Melotte 66 & 0.14 & $1,17,18$ & 13.0 & 10,11 & 6.3 & $9-11,19$ & -0.51 & $1,17,18$ & 259.6 & -14.3 & 9.9 \\
\hline M67 & 0.03 & 4,31 & 9.6 & $5,7,9-12$ & 4.2 & $3-12,16$ & -0.09 & $1-4,8$ & 215.6 & +31.7 & 9.1 \\
\hline NGC 2204 & 0.08 & $1,20,21$ & 13.1 & 10,22 & 2.8 & 10,22 & -0.38 & $1,20,21$ & 226.0 & -16.1 & 11.6 \\
\hline NGC 2243 & 0.04 & $1,23,24$ & 12.9 & $9-11,30$ & 4.4 & $8-11,30$ & -0.63 & $1,8,23$ & 239.5 & -18.0 & 10.8 \\
\hline NGC 2420 & 0.02 & $1,23,25$ & 11.8 & $9-15$ & 3.9 & $8-16$ & -0.45 & $1,8,25$ & 198.1 & +19.7 & 10.6 \\
\hline NGC 2477 & $0.22^{d}$ & text & 10.5 & 1 & 1.0 & 8,26 & -0.02 & $1,8,26$ & 253.6 & -5.8 & 8.9 \\
\hline NGC 2506 & 0.05 & $1,8,16$ & 12.2 & 10,16 & 3.5 & $8-10,16$ & -0.52 & $1,8,16$ & 230.6 & +9.9 & 10.4 \\
\hline NGC 2660 & 0.38 & 27 & 12.3 & 28 & 0.8 & 28 & -0.19 & 1,29 & 265.9 & +3.0 & 9.2 \\
\hline
\end{tabular}

an Gyr.

b Galactic coordinates in degrees.

'Galactocentric distance in kpc, assuming $R_{\odot}=8.5 \mathrm{kpc}$. in Table 7.

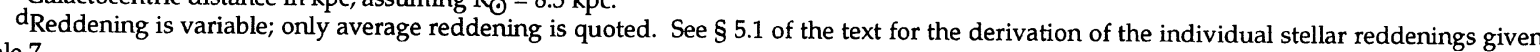

Sources: (1) Janes (1979). (2) Janes \& Smith (1984). (3) Anthony-Twarog (1987). (4) Nissen et al. (1987). (5) Francic (1989). (6) Twarog \& Anthony-Twarog (1989). (7) Twarog (1978). (8) Hirshfeld et al. (1978). (9) Norris \& Green (1989). (10) van den Bergh \& McClure (1980). (11) Gratton (1982). (12) VandenBerg (1985). (13) McClure et al. (1978). (14) Christian et al. (1985). (15) Anthony-Twarog et al. (1990). (16) McClure et al. (1981). (17) Hawarden (1976b). (18) Dawson (1978). (19) Anthony-Twarog et al. (1979). (20) Hawarden (1976a). (21) Dawson (1981). (22) Frogel \& Twarog (1983). (23) Hawarden (1975). (24) van den Bergh (1977). (25) McClure et al. (1974). (26) Smith \& Hesser (1983). (27) Hartwick \& Hesser (1973). (28) Mazzei \& Pigatto (1988). (29) Hesser \& Smith (1987). (30) Bonifazi et al. (1990). (31) Burstein et al. (1986). 
and $\tau$, no attempt was made to adjust their reported values for differences in reddening and isochrone metallicity used by various authors. Although a compelling body of evidence is accumulating in support of isochrones which include convective overshooting (e.g., Maeder \& Meynet 1989 and Bertelli et al. 1985 discuss the implications of the evolutionary models; Mazzei \& Pigatto 1988 and Anthony-Twarog et al. 1990 apply the isochrones to open clusters), the set of available overshoot isochrones span only a small range in $[\mathrm{Fe} / \mathrm{H}]$ and have not been fit to the CMDs of most of the clusters we observed. Thus, consistency demanded that we not include ages and distance moduli derived from overshoot isochrones in the cluster averages.

In the case of the distance modulus determinations, evolutionary deviation diagrams and zero-age main-sequence (ZAMS)-fitting techniques were considered inherently less reliable than isochrone predictions due to uncertainties in the Hyades modulus and the influence of metallicity upon the ZAMS position. In most cases, however, the modulus we chose agrees quite well with the ZAMS-fitting result of Janes (1979), which appears to be the most complete of the derivations that do not incorporate isochrones. For this reason, $(m-M)_{0}$ was taken from Janes for NGC 2477 because no isochrone-fitting modulus was available; however, the variable reddening of the cluster (see Sec. 4.6) may not have been taken into account in Janes' ZAMS fit.

\subsection{Metallicities}

For the sake of consistency and because of a lack of spectrophotometry for most of the clusters studied here, metallicities were estimated by averaging photometric determinations based upon the $u v b y, U B V$, or DDO system. Since the photometric metallicity estimates $[\delta(U-B)$ or $\delta(\mathrm{CN})]$ generally agreed between authors, we included only sources which directly converted their measured photometric parameter to $[\mathrm{Fe} / \mathrm{H}]$. Each of these sources was considered separately, even though one author may simply have provided a reanalysis of another's photometry. If a single reference reported more than one metallicity determination, the average of its determinations was first found, and this value was then used in computing the overall cluster average. In addition, the zero point of each particular calibration was adjusted to give $[\mathrm{Fe} / \mathrm{H}]=0.10$ for the Hyades, and the reported metallicities were revised accordingly.

In general, there is only a range of about 0.1 dex in the $[\mathrm{Fe} / \mathrm{H}]$ measurements for a given cluster; however, Hesser \& Smith's (1987) DDO photometry indicates that NGC 2660 is much more metal poor than previously thought. Because their reported metallicity is derived by excluding a star in our sample, we have revised their value of $[\mathrm{Fe} / \mathrm{H}]$ to include all of the stars they observed; the cluster average incorporates this revised metallicity, which still is considerably below solar. Further study of the NGC 2660 giants is clearly required.

The metallicities given in Table 1 agree quite well with spectrophotometric averages in those clusters for which such information was found (M67 and NGC 2420); photographic spectra, on the other hand, tend to indicate that the clusters are more metal poor than the values given in Table 1.

\subsection{Membership}

All stars are initially considered to be cluster members unless they have a membership probability less than $50 \%$ in at least one proper motion study. Other potential criteria (e.g., radial velocity, position in a CMD) which may suggest that particular stars are nonmembers are discussed in Sec. 4. Unfortunately, only three of the clusters we observed have published proper motion determinations. We indicate below the proper motion nonmembers included in our observing lists but subsequently excluded from the analysis; stars with membership probabilities between $50 \%$ and $90 \%$ are noted.

M67. Only Sanders (1977) derives proper motions for all of the stars in Table 3 . He finds membership probabilities less than $50 \%$ for stars T626 and T829; the following stars may also be suspect: IV-20 (64\%), T654 (77\%), T856 (82\%), T1189 (77\%), and IV-202 (51\%). All of the other cluster stars we observed have membership probabilities $>90 \%$ in Sanders' study. Recent proper motion work by Girard et al. (1989) and by Francic (1989) only includes stars F84 through IV-81 in Table 3 . All of these stars have proper motion membership probabilities $>98 \%$ in each study.

NGC 2420. Stars X and II-3-44 are proper motion nonmembers (van Altena \& Jones 1970), while the following giants have membership probabilities less than $90 \%$ : stars A (88\%), D (83\%), Q (57\%), U (89\%), and b (74\%). Van Altena \& Jones do not provide a proper motion measurement for star B, but it is classified as a probable proper motion member by Cannon \& Lloyd (1970). Star F presents somewhat of a quandary. Van Altena \& Jones assign this star a $90 \%$ membership probability, while Cannon \& Lloyd are ambiguous about its membership; its proper motion does not meet their membership criterion, but it is included in their CMD of probable cluster members. Because an updated analysis of the Cannon \& Lloyd data by Cabrera-Caño \& Alfaro (1990) concludes that star F is a cluster member, we have included it in our analysis. For all of the other stars in Table 6, Cabrera-Caño \& Alfaro are in agreement with Cannon \& Lloyd, excluding only star $\mathrm{X}$ from proper motion membership.

NGC 2506. Chiu \& van Altena (1981) have published the only proper motion work for this cluster. They find that stars 3254 and 4228 are not members, while the probabilities of proper motion membership for stars 1112 and 4240 are $70 \%$ and $79 \%$, respectively. Stars 2122,2212 , and 4205 are all classified as proper motion members at greater than $90 \%$ probability, while stars 2401,2402 , and 4402 are not included in this proper motion study. We note that Mathieu \& Latham (1990) question the nonmembership of star 3254 because its radial velocity agrees with the high radial velocity $(\sim 80 \mathrm{~km} / \mathrm{s})$ of the cluster itself. Nevertheless, we do not include it as a member of NGC 2506.

\section{THE INFRARED OBSERVATIONS}

The infrared observations of the cluster giants presented in this paper were obtained on the 4 and $1.5 \mathrm{~m}$ telescopes at CTIO and the $2.5 \mathrm{~m}$ du Pont telescope at Las Campanas Observatory. These data were obtained and reduced in an identical manner and generally on the same nights as the data for the globular cluster program described in FPC83. Table 1 of FPC83 shows that there are no statistically significant differences between datasets obtained at the two observatories.

Most of the brighter giants evident from optical CMDs were selected for observation in the infrared. The new infrared photometry is presented in Tables 2-9; most of the data for M67 were previously published in CFP. The stars with proper motion membership probabilities less than $50 \%$ 
have been included in the data tables but are omitted from the figures. For all of the photometry presented here, the reddening ratios of Cohen et al. (1981) have been applied; we assume that the cluster giants are spectral type $\mathrm{K} 0$ and linearly interpolate Cohen et al.'s Table 10 to find the proper ratios. Also, we take $\mathrm{E}(U-V)=1.71 \mathrm{E}(B-V)$.

\section{THE INFRARED COLOR-MAGNITUDE DIAGRAMS}

$M_{K},(V-K)_{0}$ color-magnitude diagrams of the eight open clusters examined here are shown in Figs. 1-8. These can be compared to CMDs based upon optical photometry to help define the locus of the cluster GB and identify possible AGB, anomalous, or nonmember stars. To properly examine the cluster CMDs, occasional reference will have to be made to the color-color plots and CO indices (Figs. 9-12) that are discussed in detail in Sec. 5. Symbols bracketed by parentheses in Figs. 1-8 represent stars which may be nonmembers on the basis of radial velocity data; such indications are discussed in the respective cluster's subsection of the following text.

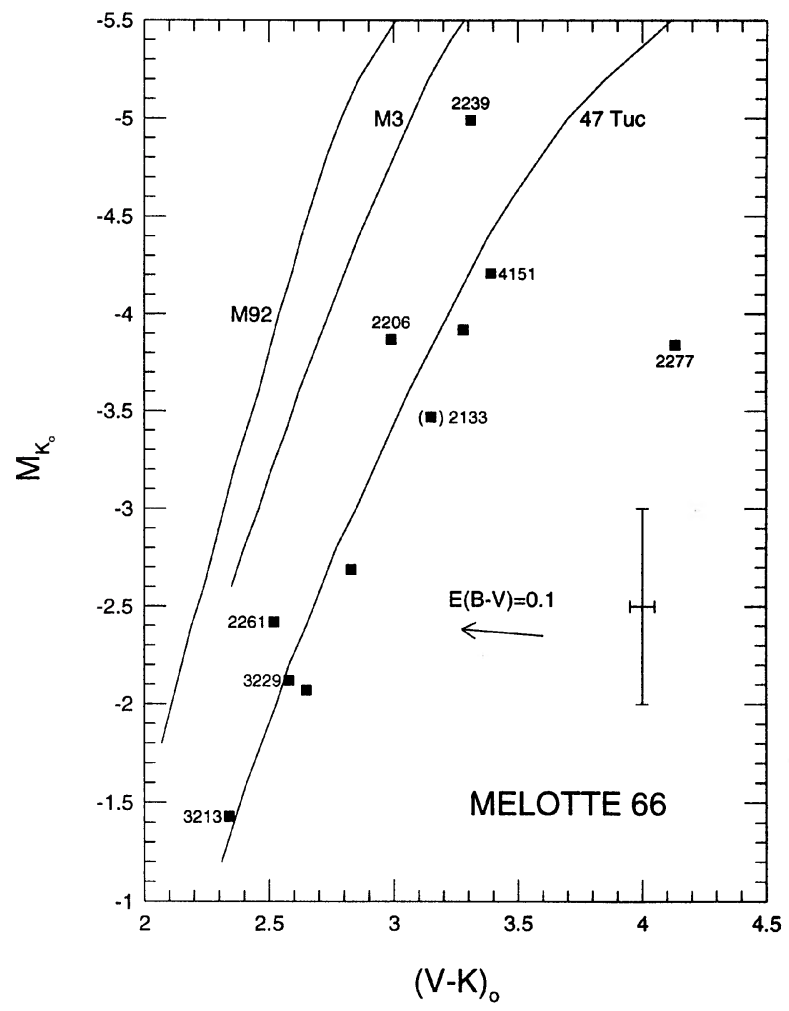

FIG. 1. Infrared CMD of Melotte 66. Star 2215 is not shown; it lies on a red extension of the GB at $M_{K}=-6.92,(V-K)_{0}=6.77$. Interesting stars discussed in the text are individually labeled; symbols enclosed in parentheses represent radial velocity nonmembers. The GBs of the globular clusters M3, M92, and 47 Tucanae (taken from Table 5 of FPC81) are presented for comparison. The error bars represent the uncertainties of $\pm 0.05 \mathrm{mag}$ in $V$ $K$ and $\pm 0.50 \mathrm{mag}$ in $(m-M)_{0}$ given in Sec. 2 ; the effect of increasing the value of $\mathrm{E}(B-V)$ by $0.1 \mathrm{mag}$ is also shown. In this and all succeeding figures, consistent use is made of the specific symbol used to represent the stars from a particular cluster.

\subsection{Melotte 66}

Infrared data for Melotte 66 are given in Table 2(a); its infrared CMD is shown in Fig. 1. In general appearance, the CMD agrees with the optical diagrams of Hawarden (1976b) and Anthony-Twarog et al. (1979, hereafter referred to as ATM) in presenting the appearance of either an inherently broad GB or a well-defined GB with a few AGB stars. However, the possibility that a significant fraction of the giants are spectroscopic binaries cannot be ruled out by any radial velocity data published to date for Melotte 66. In fact, recent radial velocity studies of young and intermediate age open clusters (e.g., Levato et al. 1990; Mermilliod \& Mayor 1989, 1990) reveal that $25 \%-33 \%$ of the stars observed by these authors are spectroscopic binaries. It is surprising that no complete proper motion or radial velocity studies have been performed for Melotte 66; its GB morphology could contain valuable information regarding binary stars in open clusters or provide a template for distinguishing other open cluster AGB stars.

If an AGB is present, it is defined by stars 2206, 2239, and $2261 ; 3213$ is probably a clump star. Suspected abundance peculiarities in stars 2239 and 2261 (Hawarden 1976b) require further observation; no CO measurement was made for 2261, while 2239 appears to have CO appropriate to other members of the cluster [see Fig. 12(b) ]. Although star 2261 seems to deviate from the cluster GB star trend in Fig. 9(b) (and 2239 may also), there are too few GB stars in Melotte 66 with $U$ photometry to determine the cluster locus definitively in this diagram. Star 3229, meanwhile, appears to be an AGB star in Hawarden's optical CMD but lies closer to the GB in that of ATM and in the infrared. The range of $V$ magnitudes reported for 3229 is only $0.07 \mathrm{mag}$, so it is likely not a variable; its low $\mathrm{CO}$ value could be a consequence of photometric uncertainties or may indicate a true chemical peculiarity.

Four other giants in Melotte 66 merit discussion. Star 2277, to the red of the GB in Fig. 1, may be variable, as suspected by Hawarden (1976b); his photographic and photoelectric photometry, combined with the photographic values of ATM, however, only show a range of $0.06 \mathrm{mag}$ in $V$ and $0.07 \mathrm{mag}$ in $B-V$. Star 2215 (not shown in Fig. 1) lies on a reasonable extension of the GB in the infrared, but it lies well to the red of the optical GB. The 0.22 mag difference between the photographic $V$ magnitudes reported by Hawarden and ATM may indicate that this star is also variable; the infrared data in Table 2(b), though, appear to rule out large amplitude variability. Geisler \& Smith (1984) claim that star 2133 may be a radial velocity nonmember but do not provide the specific data they use to reach this conclusion; it lies on the GB in Fig. 1, but its CO value is relatively low for the cluster [see Fig. 12(b)]. Star 4151, on the other hand, exhibits high $\mathrm{CO}$ and a slightly high $\delta \mathrm{CN}$ (Dawson 1978 ), but it also clearly lies on the GB in both the optical and the infrared CMDs.

\subsection{M67 (NGC 2682)}

Infrared data for stars in M67 are given in Table 3; most of this photometry was previously published in CFP, but it is included here for completeness. The infrared GB of this cluster, shown in Fig. 2, is well defined and nearly identical in appearance to its optical counterparts in Johnson \& Sandage (1955), Eggen \& Sandage (1964), Racine (1971), and Janes \& Smith (1984). 
TABLE 2(a). Melotte 66 photometry.

\begin{tabular}{|c|c|c|c|c|c|c|c|c|c|c|c|c|c|}
\hline \multirow{3}{*}{$\frac{S^{\text {Stara }}}{2133}$} & \multicolumn{3}{|c|}{ Observed $^{b}$} & \multicolumn{8}{|c|}{ Reddening Corrected ${ }^{c}$} & \multirow{2}{*}{$\mathrm{n}$} & \multirow{2}{*}{ Notes } \\
\hline & \multirow{2}{*}{$\begin{array}{c}K \\
9.58\end{array}$} & \multirow{2}{*}{$\frac{J-K}{0.91}$} & \multirow{2}{*}{$\frac{H-K}{0.17}$} & \multirow{2}{*}{$\frac{K_{\mathrm{o}}}{9.53}$} & \multicolumn{5}{|c|}{$(U-V)_{0}(B-V)_{0}(V-K)_{0}(J-K)_{0}(H-K)_{0}$} & \multirow{2}{*}{$\frac{\mathrm{H}_{2} \mathrm{O}}{\ldots}$} & \multirow{2}{*}{$\frac{\mathrm{CO}}{0.105}$} & & \\
\hline & & & & & 2.83 & 1.36 & 3.15 & 0.82 & 0.14 & & & 1 & 3,7 \\
\hline 4151 & 8.84 & 0.95 & 0.18 & 8.79 & $\ldots$ & 1.43 & 3.39 & 0.86 & 0.15 & $\ldots$ & 0.155 & 1 & 1 \\
\hline 1242 & 9.13 & 0.95 & 0.17 & 9.08 & $\ldots$ & 1.42 & 3.28 & 0.86 & 0.14 & $0.01 \quad(3)$ & 0.135 & 1 & 6 \\
\hline 2206 & 9.18 & 0.89 & 0.16 & 9.13 & $\ldots$ & 1.32 & 2.99 & 0.80 & 0.13 & 0.025 & 0.115 & 2 & 2 \\
\hline 2215 & $6.13(3)$ & 1.26 & 0.31 & 6.08 & $\ldots$ & 1.59 & 6.77 & 1.17 & 0.28 & 0.17 (3) & 0.205 & 4 & 6,8 \\
\hline 2217 & 10.98 & 0.78 & 0.14 & 10.93 & 2.25 & 1.13 & 2.65 & 0.69 & 0.11 & $\ldots$ & 0.08 & 1 & 5,7 \\
\hline 2239 & 8.06 & 0.93 & 0.17 & 8.01 & 3.18 & 1.44 & 3.31 & 0.84 & 0.14 & 0.03 & 0.13 & 1 & 4,7 \\
\hline 2261 & 10.63 & 0.73 & 0.15 & 10.58 & 2.27 & 1.11 & 2.52 & 0.64 & 0.12 & $\ldots$ & $\ldots$ & 1 & 3,7 \\
\hline 2277 & 9.21 & 1.08 & 0.23 & 9.16 & $\ldots$ & 1.70 & 4.13 & 0.99 & 0.20 & 0.045 & 0.155 & 1 & 1 \\
\hline 3213 & $11.62(3)$ & $0.68(3)$ & $0.16(3)$ & 11.57 & 1.82 & 1.01 & 2.34 & 0.59 & 0.13 & ... & ... & 1 & 1,7 \\
\hline 3229 & 10.93 & 0.73 & 0.16 & 10.88 & ... & 1.11 & 2.58 & 0.64 & 0.13 & ... & 0.03 & 1 & 2 \\
\hline 4229 & 10.36 & 0.84 & 0.15 & 10.31 & $\ldots$ & 1.23 & 2.83 & 0.75 & 0.12 & $\ldots$ & 0.095 & 1 & 6 \\
\hline
\end{tabular}

aStar designations are from Hawarden (1976b).

bObservational uncertainties in the infrared magnitudes and colors are \pm 0.02 mag unless otherwise noted (in units of hundredths of a magnitude in parentheses).

CReddening ratios are those of a K0 star as given by Cohen et al. (1981).

Notes: (1) Photoelectric $B V$ photometry from Hawarden (1976b). (2) Photoelectric $B V$ photometry from Hawarden (1978). (3) Photoelectric $B V$ photometry from Hawarden (1976b) and Geisler \& Smith (1984). (4) Photoelectric $B V$ photometry from Hawarden (1976b, 1978) and Geisler \& Smith (1984). (5) Photoelectric BV photometry from Hawarden (1976b, 1978), Anthony-Twarog et al. (1979) and Geisler \& Smith (1984). (6) Photographic BV photometry from Hawarden (1976b) and Anthony-Twarog et al. (1979). (7) Photoelectric $U-B$ color from Hawarden (1976b). (8) Possible red variable; range in $V$ is $\sim 0.2$ mag. Infrared photometry is average of values in Table $2 \mathrm{~b} ;(K-L)_{0}=0.23$.

TABLE 2(b). Photometry of star 2215 in Melotte 66.

\begin{tabular}{ccccccc}
\hline \hline Date & \multicolumn{7}{c}{ Observeda $^{\mathrm{c}}$} \\
\cline { 2 - 7 } JD2440000+ & $K$ & $J-K$ & $H-K$ & $K-L$ & $\mathrm{H}_{2} \mathrm{O}$ & $\mathrm{CO}$ \\
\hline 3900 & 6.16 & 1.27 & 0.31 & 0.23 & 0.215 & 0.21 \\
3916 & 6.13 & 1.27 & 0.31 & $\ldots$ & 0.19 & 0.19 \\
3946 & 6.14 & 1.26 & 0.31 & 0.26 & 0.16 & 0.195 \\
3970 & 6.09 & 1.24 & 0.30 & 0.27 & 0.16 & 0.195 \\
\hline
\end{tabular}

aObservational uncertainties in the infrared magnitudes and colors are \pm 0.02 mag.

Cohen (1980), Mathieu et al. (1986), Pilachowski et al. (1988), and Friel et al. (1989) have published radial velocities for subsets of the stars in Table 3. Cohen's spectra of stars F105, F170, F224, and F231 indicate that all four are cluster members. Similarly, Friel et al. assign radial velocity membership to each of the stars from Table 3 which they observed - F84, F141, and F170. Pilachowski et al. find that nine of the eleven giants they studied (F84, F105, F108, F141, F170, T626, T829, T856, and T1189) are cluster members, while they classify stars T574 and T654 as field interlopers. However, the most complete radial velocity study of M67 is that of Mathieu et al. They observed all of the stars in Table 3 except F94 and conclude that only stars T574 and T654 are nonmembers, in agreement with Pilachowski et al. Although this supports the claim of Janes \& Smith (1984) that T574 is either a binary, AGB star, or nonmember, both T574 and T654 are included in our analysis on the basis of the aforementioned proper motion work (see Sec. 2.5). However, their positions in color-color diagrams [see Fig. 9(a) for T574 and Fig. 10(a) for T654] could also be consistent with nonmembership.

There are no obvious AGB stars in M67. Stars F84, F141, $F 151, F 164$, and F223 are clump stars. Although F224 lies in the region of the clump, it is a long period ( $\sim 6000$ day) spectroscopic binary (SB) according to Mathieu et al. (1986) and Mathieu \& Latham (1986). Mathieu et al. find that stars F117 and F244 are also SBs. This explains the variability of F117 noted by Schild (1983) and the questions 
TABLE 3. M67 (NGC 2682) photometry.

\begin{tabular}{|c|c|c|c|c|c|c|c|c|c|c|c|c|c|}
\hline \multirow{2}{*}{ Stara } & \multicolumn{3}{|c|}{ Observed ${ }^{b}$} & \multicolumn{8}{|c|}{ Reddening Correctedc } & \multirow{2}{*}{$\mathrm{n}$} & \multirow{2}{*}{ Notes } \\
\hline & $K$ & $J-K$ & $H-K$ & $K_{\mathrm{o}}$ & $(U-V)_{C}$ & $B-V)_{C}$ & $V-K)_{C}$ & $(J-K)_{C}$ & $H-K)_{0}$ & $\mathrm{H}_{2} \mathrm{O}$ & $\mathrm{CO}$ & & \\
\hline F84 & $8.10(4)$ & $0.63(4)$ & $0.09(4)$ & 8.09 & 2.01 & 1.07 & 2.35 & 0.61 & 0.08 & $\ldots$ & 0.08 & 1 & 3 \\
\hline F94 & 11.44 & 0.34 & 0.06 & 11.43 & 0.58 & 0.53 & 1.29 & 0.32 & 0.05 & $\ldots$ & 0.03 & 1 & 1 \\
\hline F105 & 7.47 & 0.75 & 0.10 & 7.46 & 2.55 & 1.23 & 2.73 & 0.73 & 0.09 & 0.085 & 0.13 & 1 & 4 \\
\hline F108 & 6.57 & 0.80 & 0.11 & 6.56 & 2.86 & 1.33 & 3.04 & 0.78 & 0.10 & 0.05 & 0.11 & 1 & 5 \\
\hline F115 & 11.17 & 0.36 & 0.05 & 11.16 & 0.70 & 0.60 & 1.38 & 0.34 & 0.04 & $\ldots$ & 0.015 & 1 & 1 \\
\hline F117 & 10.58 & 0.55 & 0.08 & 10.57 & 1.00 & 0.75 & 1.98 & 0.53 & 0.07 & $\ldots$ & 0.055 & 1 & 6 \\
\hline F141 & $7.99(4)$ & $0.63(4)$ & 0.09 (4) & 7.98 & 2.04 & 1.08 & 2.38 & 0.61 & 0.08 & $\ldots$ & 0.07 & 1 & 7 \\
\hline F151 & $8.06(4)$ & $0.62(4)$ & 0.09 (4) & 8.05 & 2.00 & 1.06 & 2.33 & 0.60 & 0.08 & $\ldots$ & 0.07 & 1 & 7 \\
\hline F164 & 8.01 & 0.63 & 0.09 & 8.00 & 2.09 & 1.09 & 2.41 & 0.61 & 0.08 & 0.01 & 0.06 & 1 & 8 \\
\hline F170 & 6.60 & 0.78 & 0.11 & 6.59 & 2.78 & 1.32 & 2.98 & 0.76 & 0.10 & 0.04 & 0.13 & 1 & 8 \\
\hline F193 & $9.96(4)$ & $0.58(4)$ & $0.07(4)$ & 9.95 & 1.77 & 0.99 & 2.22 & 0.56 & 0.06 & $\ldots$ & $\ldots$ & 1 & 9 \\
\hline F223 & $8.04(4)$ & $0.66(4)$ & $0.11(4)$ & 8.03 & 2.04 & 1.08 & 2.42 & 0.64 & 0.10 & $\ldots$ & 0.035 & 1 & 10 \\
\hline F224 & 8.23 & 0.62 & 0.08 & 8.22 & 2.15 & 1.08 & 2.44 & 0.60 & 0.07 & 0.04 & 0.07 & 1 & 7 \\
\hline F227 & $10.82(5)$ & $0.59(5)$ & 0.14 (5) & 10.81 & 1.46 & 0.88 & 2.06 & 0.57 & 0.13 & $\ldots$ & $\ldots$ & 1 & 11 \\
\hline F231 & $9.11(4)$ & $0.64(4)$ & $0.08(4)$ & 9.10 & 1.88 & 1.02 & 2.30 & 0.62 & 0.07 & $\ldots$ & ... & 1 & 10 \\
\hline F244 & $8.60(4)$ & $0.56(4)$ & $0.10(4)$ & 8.59 & 1.56 & 0.91 & 2.07 & 0.54 & 0.09 & $\ldots$ & 0.04 & 1 & 12 \\
\hline I-17 & 10.09 & 0.56 & 0.10 & 10.08 & 1.67 & 0.96 & 2.21 & 0.54 & 0.09 & $\ldots$ & 0.04 & 1 & 1 \\
\hline II-22 & 10.76 & 0.50 & 0.07 & 10.75 & 1.47 & 0.89 & 2.08 & 0.48 & 0.06 & $\ldots$ & 0.05 & 1 & 11 \\
\hline III-34 & 8.77 & 0.60 & 0.07 & 8.76 & 1.95 & 1.06 & 2.45 & 0.58 & 0.06 & $\ldots$ & 0.075 & 1 & 1 \\
\hline IV-20 & 8.60 & 0.63 & 0.08 & 8.59 & 1.91 & 1.04 & 2.51 & 0.61 & 0.07 & $\ldots$ & 0.065 & 1 & 1 \\
\hline IV-68 & 10.84 & 0.55 & 0.12 & 10.83 & 1.30 & 0.84 & 2.01 & 0.53 & 0.11 & $\ldots$ & 0.025 & 1 & 1 \\
\hline IV-77 & 10.66 & 0.53 & 0.07 & 10.65 & 1.58 & 0.95 & 2.13 & 0.51 & 0.06 & $\ldots$ & 0.05 & 1 & 1 \\
\hline IV-81 & 10.89 & 0.44 & 0.06 & 10.88 & 1.15 & 0.79 & 1.80 & 0.42 & 0.05 & $\ldots$ & 0.03 & 1 & 1 \\
\hline T574 & 7.11 & 0.74 & 0.12 & 7.10 & 2.42 & 1.20 & 2.81 & 0.72 & 0.11 & 0.07 & 0.105 & 1 & 2 \\
\hline T626 & 5.95 & 0.87 & 0.16 & 5.94 & 3.19 & 1.44 & 3.32 & 0.85 & 0.15 & 0.04 & 0.135 & 4 & 13,14 \\
\hline T654 & $7.82(3)$ & 0.65 & 0.12 & 7.81 & 2.48 & 1.20 & 2.60 & 0.63 & 0.11 & 0.01 & 0.09 & 1 & 2 \\
\hline T829 & 6.45 & 0.78 & 0.13 & 6.44 & 2.90 & 1.35 & 2.98 & 0.76 & 0.12 & 0.075 & 0.125 & 1 & 2,15 \\
\hline T856 & 6.78 & $0.78(3)$ & 0.11 & 6.77 & 2.79 & 1.33 & 2.96 & 0.76 & 0.10 & 0.065 & 0.105 & 1 & 2 \\
\hline T1189 & 7.13 & 0.78 & 0.19 & 7.12 & 2.71 & 1.27 & 2.89 & 0.76 & 0.18 & 0.06 & 0.10 & 1 & 2 \\
\hline IV-202 & 5.08 & 0.91 & 0.17 & 5.07 & 3.48 & 1.54 & 3.66 & 0.89 & 0.16 & 0.05 & 0.16 & 2 & 13,16 \\
\hline
\end{tabular}

aStar designations are from Fagerholm (1906) when preceded by F, Eggen \& Sandage (1964) when preceded by a Roman numeral and Murray \& Clements (1968) when preceded by T.

bObservational uncertainties in the infrared magnitudes and colors are \pm 0.02 mag unless otherwise noted (in units of hundredths of a magnitude in parentheses).

'Reddening ratios are those of a K0 star as given by Cohen et al. (1981).

Notes: (1) Photoelectric UBV photometry from Eggen \& Sandage (1964, hereafter ES). (2) Photoelectric UBV photometry from Janes \& Smith (1984). (3) Photoelectric UBV photometry from ES, Sturch (1973) and Eggen (1983). (4) Photoelectric UBV photometry from ES, Sturch (1972, 1973), Coleman (1982) and Eggen (1983). (5) Photoelectric UBV photometry from ES, Coleman (1982), Schild (1983) and Eggen (1983). (6) Photoelectric UBV photometry from ES, Sturch (1973) and Schild (1983). (7) Photoelectric UBV photometry from ES, Sturch (1973), Coleman (1982) and Eggen (1983). (8) Photoelectric UBV photometry from ES, Coleman (1982) and Eggen (1983). (9) Photoelectric UBV photometry from ES and Eggen (1983). (10) Photoelectric UBV photometry from ES and Coleman (1982). (11) Photoelectric $U B V$ photometry from ES and Sturch (1972, 1973). (12) Photoelectric UBV photometry from ES, Sturch (1973) and Coleman (1982). (13) Photoelectric UBV photometry from Eggen (1972a, 1983) and Janes \& Smith (1984). (14) Proper motion non-member (Sanders 1977); not included in figures. $(K-L)_{0}=0.10 \pm 0.03$. (15) Same as note 14, but $(K-L)_{0}=0.09 \pm$ 0.03. (16) $(K-L)_{0}=0.10$. 


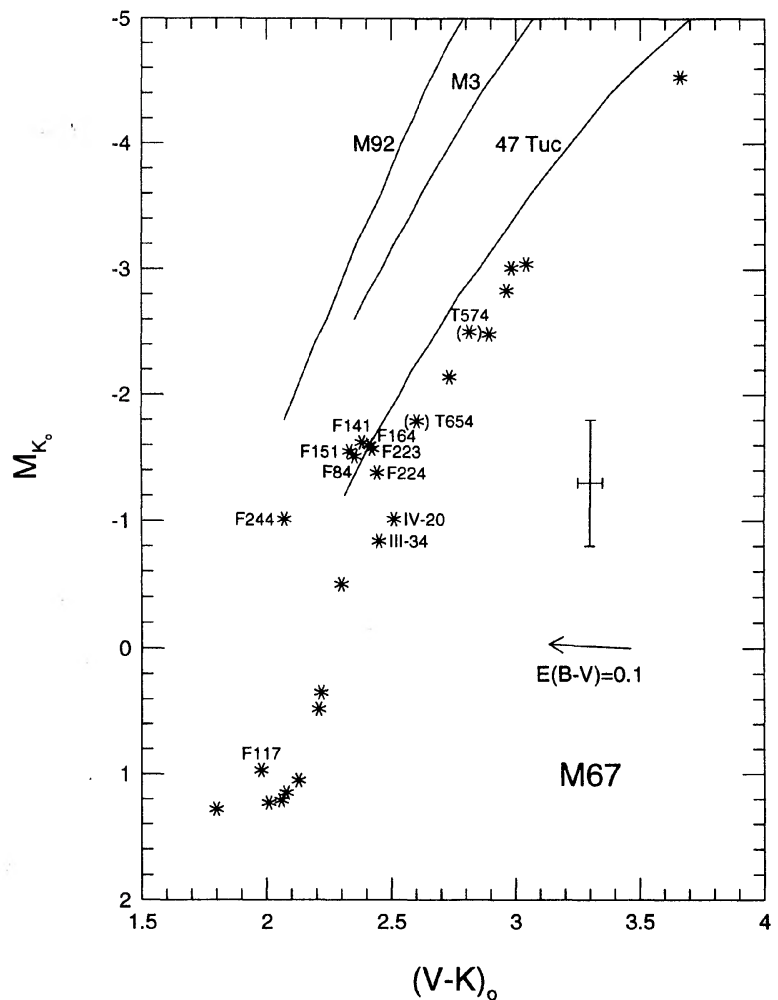

FIG. 2. Infrared CMD of M67. Stars F94 and F115 are not shown; they lie on the subgiant branch near the main sequence turnoff at $M_{K}=+1.83$, $(V-K)_{0}=1.29$ and $M_{K}=+1.56,(V-K)_{0}=1.38$, respectively. See the caption of Fig. 1 for further details.

posed regarding F244 by Janes (1974), Osborn (1974), Foy \& Proust (1981), and Coleman (1982). Foy \& Proust, however, claim that Murray et al. (1965) give star F244 less than a 5\% membership probability based upon their proper motion measurements; a review of these proper motions contradicts this claim. In addition, star F170 shows signs of possible duplicity; its radial velocity has a high standard deviation in Mathieu et al.'s study and differs from the cluster mean of Pilachowski et al. (1988) by much more than any of the other members they observed.

Several possibly anomalous stars in M67 have been cited in the literature. The enhanced $\mathrm{CN}$ reported for stars F84, F141, and F151 (Pagel 1974) and T654 (Janes \& Smith 1984 ) is not apparent in our CO measurements [see Fig. $12(\mathrm{~d})$ ]. Overall, there is little scatter in the CO plot; this makes it difficult to ascertain if any of the M67 stars observed are peculiar, but stars F223 (low CO) and F105 (high CO) may be worth further investigation. Stars III-34 and IV-20 are interesting in that they lie on the optical GB but are situated slightly to the red of the infrared GB. These stars also appear to be displaced from the mean of the other M67 giants in the color-color plots [see Figs. 9(a) and 10 (a) ], but the photographic $V$ photometry presented by Francic (1989) is sufficiently discrepant from the other reported $V$ magnitudes of these stars that photometric errors or variability are plausible for stars III-34 and IV-20.

Finally, a few stars in M67 appear to scatter around the blue end of the field line in the $(J-K)_{0},(V-K)_{0}$ plot [Fig. 10 (a) ]; these stars are all either known binaries, lie at the base of the giant branch and/or have infrared photometric uncertainties greater than the error bars shown.

\subsection{NGC 2204}

The infrared photometry for giants in NGC 2204 is given in Table 4(a); additional observations of suspected variables are presented in Tables 4(b) and 4(c). Figure 3 is the infrared CMD. Dawson (1981) uses the $B V$ CMD of Hawarden (1976a) to delineate a GB for this cluster which appears to be too red. Our infrared data suggest that his choice of GB is influenced by some probable field stars lying to the red of the true GB; this group includes stars 2222 and 2227 in our sample. Dawson's DDO photometry indicates that star 2222 is metal enhanced, but no CO measurement was made for it; the $\mathrm{CO}$ absorption for 2227 is typical of the other cluster giants in Fig. 12(a).

Star 1329, which lies to the blue of the giant branch in Fig. 3 , appears to be a nonmember of NGC 2204 on the basis of the radial velocity study by Friel (1989). In addition, our CO measurement [see Fig. 12(a) ], Dawson's (1981) DDO photometry, and Hawarden's (1976a) UBV color-color plot all show that it possesses a higher metal content than other cluster stars. 1329 also deviates from the cluster norm in the $(U-V)_{0},(V-K)_{0}$ diagram [Fig. 9(a)].

Stars 2120 and 2212 are also included in Friel's (1989) derivation of an average cluster radial velocity of $59 \mathrm{~km} / \mathrm{s}$,

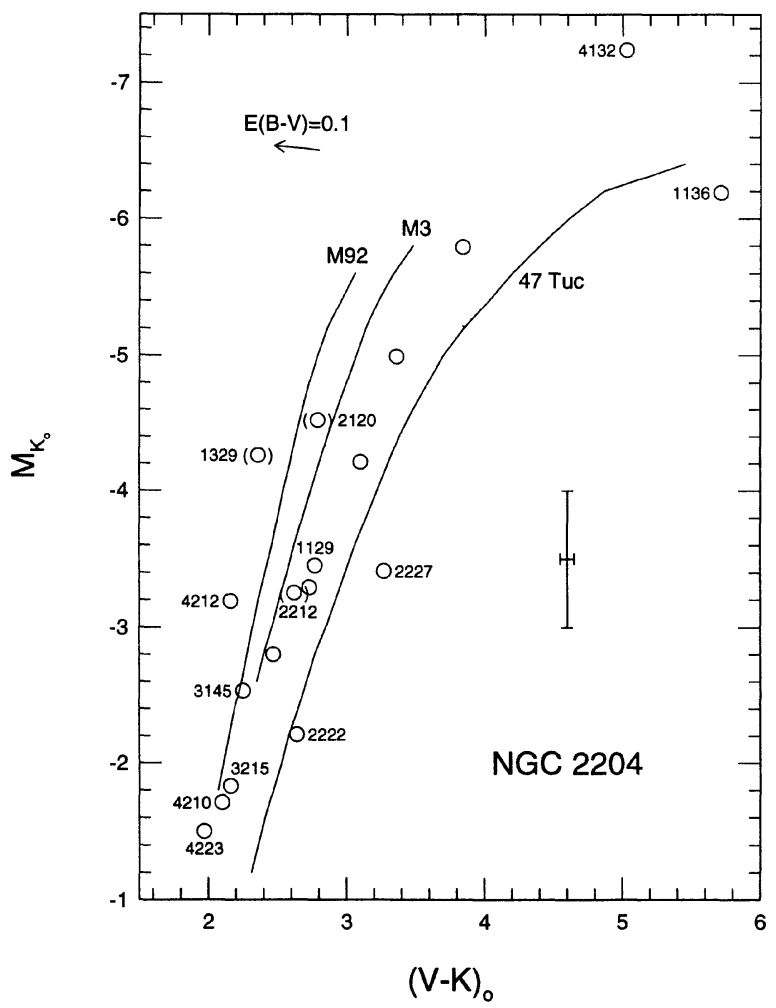

Fig. 3. Infrared CMD of NGC 2204. See the caption of Fig. 1 for further details. 
TABLE 4(a). NGC 2204 photometry.

\begin{tabular}{|c|c|c|c|c|c|c|c|c|c|c|c|c|c|}
\hline \multirow{3}{*}{$\begin{array}{l}\text { Star }^{a} \\
1129\end{array}$} & \multicolumn{3}{|c|}{ Observed $^{b}$} & \multicolumn{8}{|c|}{ Reddening Corrected ${ }^{c}$} & \multirow{2}{*}{$\mathrm{n}$} & \multirow{2}{*}{ Notes } \\
\hline & \multirow{2}{*}{$\frac{K}{9.68}$} & \multirow{2}{*}{$\frac{J-K}{0.72(3)}$} & \multirow{2}{*}{$\frac{H-K}{0.12}$} & \multirow{2}{*}{$\frac{K_{\mathrm{o}}}{9.65}$} & \multicolumn{5}{|c|}{$(U-V)_{0}(B-V)_{\mathrm{o}}(V-K)_{\mathrm{o}}(U-K)_{\mathrm{o}}(H-K)_{\mathrm{o}}$} & \multirow{2}{*}{$\frac{\mathrm{H}_{2} \mathrm{O}}{\ldots}$} & \multirow{2}{*}{$\frac{\mathrm{CO}}{0.085}$} & & \\
\hline & & & & & $\ldots$ & 1.07 & 2.77 & 0.67 & 0.10 & & & 1 & 2 \\
\hline 1136 & $6.94(3)$ & 1.17 & 0.27 & 6.91 & $\ldots$ & 1.66 & 5.71 & 1.12 & 0.25 & $0.145(4)$ & 0.215 & 5 & 2,3 \\
\hline 2120 & 8.61 & 0.75 & 0.13 & 8.58 & 2.34 & 1.21 & 2.79 & 0.70 & 0.11 & $\ldots$ & 0.115 & 1 & 1 \\
\hline 2136 & 10.33 & 0.68 & 0.10 & 10.30 & $\ldots$ & 1.05 & 2.47 & 0.63 & 0.08 & $\ldots$ & 0.075 & 2 & 2 \\
\hline 3145 & 10.60 & 0.63 & 0.09 & 10.57 & $\ldots$ & 0.92 & 2.25 & 0.58 & 0.07 & $\ldots$ & 0.04 & 1 & 2 \\
\hline 4132 & 5.89 & 1.16 & 0.25 & 5.86 & $\ldots$ & 1.74 & 5.03 & 1.11 & 0.23 & $0.115(3)$ & 0.225 & 6 & 2,4 \\
\hline 4137 & 8.14 & 0.92 & 0.16 & 8.11 & $\ldots$ & 1.55 & 3.36 & 0.87 & 0.14 & 0.04 & 0.145 & 4 & 2 \\
\hline 2212 & 9.88 & 0.73 & 0.11 & 9.85 & $\ldots$ & 1.13 & 2.62 & 0.68 & 0.09 & $0.025(3)$ & 0.08 & 2 & 1 \\
\hline 2222 & 10.92 & 0.72 & 0.12 & 10.89 & $\ldots$ & 1.21 & 2.64 & 0.67 & 0.10 & $\ldots$ & $\ldots$ & 1 & 1 \\
\hline 2227 & 9.72 & 0.90 & 0.15 & 9.69 & $\ldots$ & 1.30 & 3.27 & 0.85 & 0.13 & 0.055 & 0.15 & 1 & 2 \\
\hline 3215 & 11.30 & 0.59 & 0.09 & 11.27 & 1.54 & 0.92 & 2.16 & 0.54 & 0.07 & $\ldots$ & 0.025 & 1 & 1 \\
\hline 4210 & 11.42 & 0.58 & 0.10 & 11.39 & 1.54 & 0.90 & 2.10 & 0.53 & 0.08 & $\ldots$ & $\ldots$ & 1 & 1 \\
\hline 4212 & 9.94 & 0.60 & 0.09 & 9.91 & $\ldots$ & 0.90 & 2.16 & 0.55 & 0.07 & $\ldots$ & 0.045 & 1 & 2 \\
\hline 4223 & 11.63 & 0.56 & 0.10 & 11.60 & 1.46 & 0.94 & 1.97 & 0.51 & 0.08 & $\ldots$ & $\ldots$ & 1 & 1 \\
\hline 1329 & 8.87 & $0.64(3)$ & 0.13 & 8.84 & 2.23 & 1.09 & 2.36 & 0.59 & 0.11 & $\ldots$ & 0.105 & 1 & 1 \\
\hline 3304 & 8.92 & 0.84 & 0.14 & 8.89 & 2.74 & 1.34 & 3.10 & 0.79 & 0.12 & $0.055(3)$ & 0.135 & 2 & 1 \\
\hline 3324 & 9.84 & 0.78 & 0.13 & 9.81 & $\ldots$ & 1.22 & 2.73 & 0.73 & 0.11 & $\ldots$ & 0.105 & 1 & 2 \\
\hline 3325 & 7.34 & $0.99(3)$ & 0.19 & 7.31 & $\ldots$ & 1.72 & 3.84 & 0.94 & 0.17 & $0.045(4)$ & 0.175 & 1 & 2 \\
\hline
\end{tabular}

aStar designations are from Hawarden (1976a).

bObservational uncertainties in the infrared magnitudes and colors are \pm 0.02 mag unless otherwise noted (in units of hundredths of a magnitude in parentheses).

'Reddening ratios are those of a K0 star as given by Cohen et al. (1981).

Notes: (1) Photoelectric UBV photometry from Hawarden (1976a). (2) Photographic BV photometry from Hawarden (1976a). (3) Probable red variable; range in $V$ is $\sim 0.4$ mag. Infrared photometry is average of values in Table $4 \mathrm{~b} ;(K-L) \mathrm{o}=0.19$. (4) Possible red variable. Infrared photometry is average of values in Table $4 c ;(K-L)_{0}=0.17 \pm 0.03$.

TABLE 4(b). Photometry of star 1136 in NGC 2204.

\begin{tabular}{ccccccl}
\hline Date & \multicolumn{7}{c}{ Observed $^{\mathrm{a}}$} \\
\cline { 2 - 7 } JD2440000+ & $K$ & $J-K$ & $H-K$ & $K-L$ & $\mathrm{H}_{2} \mathrm{O}$ & $\mathrm{CO}$ \\
\hline 3840 & 6.96 & 1.14 & 0.26 & $\ldots$ & $0.12(4)$ & 0.20 \\
3901 & 6.92 & 1.18 & 0.27 & $\ldots$ & 0.14 & 0.205 \\
3946 & 6.93 & 1.17 & 0.27 & $0.18(3)$ & $0.15(3)$ & 0.20 \\
3973 & 6.90 & 1.16 & 0.26 & $0.22(3)$ & $0.12(3)$ & 0.22 \\
3997 & 6.97 & 1.20 & 0.27 & $0.19(5)$ & $0.22(5)$ & 0.24 \\
\hline
\end{tabular}

aObservational uncertainties in the infrared magnitudes and colors are $\pm 0.02 \mathrm{mag}$ unless otherwise noted (in units of hundredths of a magnitude in parentheses). $K, J-K$ and $H-K$ for JD2443840 are averages of two observations on that date.

TABLE 4(c). Photometry of star 4132 in NGC 2204.

\begin{tabular}{cllllll}
\hline \hline Date & \multicolumn{7}{c}{ Observed $^{\mathrm{a}}$} \\
\cline { 2 - 7 } JD2440000+ & $K$ & $J-K$ & $H-K$ & $K-L$ & $\mathrm{H}_{2} \mathrm{O}$ & $\mathrm{CO}$ \\
\hline 3840 & 5.88 & $1.13(3)$ & 0.25 & $\ldots$ & $0.10(4)$ & 0.20 \\
3850 & 5.88 & 1.18 & 0.26 & $\ldots$ & 0.10 & 0.22 \\
3857 & 5.90 & $1.15(3)$ & 0.25 & $\ldots$ & 0.12 & 0.235 \\
3946 & 5.90 & 1.16 & 0.24 & $0.16(3)$ & $0.12(3)$ & 0.22 \\
3973 & 5.87 & 1.13 & 0.24 & $0.21(3)$ & $0.12(3)$ & 0.22 \\
3997 & 5.91 & 1.18 & 0.25 & $0.17(5)$ & $0.17(5)$ & 0.24 \\
\hline \hline
\end{tabular}

aObservational uncertainties in the infrared magnitudes and colors are \pm 0.02 mag unless otherwise noted (in units of hundredths of a magnitude in parentheses). 
substantially different from the $101 \mathrm{~km} / \mathrm{s}$ average measured by Cameron \& Reid (1987). Noting that only one of the six stars observed by Friel lies on the GB in Hawarden's (1976a) optical CMD (two are clump stars) and using the Cameron \& Reid data as a guide, we find that Friel's radial velocities indicate that stars 2120 and 2212 are probable nonmembers as well. However, we observe that the former star, star 3145, and star 4212, all three of which lie somewhat to the blue of the GB, show no CO anomaly in Fig. 12(a); they are possibly AGB stars. Comparison of the photographic magnitudes of 34 main-sequence stars from Hawarden (1976a) with the vidicon photometry of Frogel \& Twarog (1983) indicates that large random uncertainties may be present in the photographic data. Thus, since photographic $V$ photometry was used for stars 3145 and 4212, their colors are less certain than that of star 2120 , for which photoelectric observations were available. 2120 lies blueward of the cluster $\mathrm{GB}$ by about $0.4 \mathrm{mag}$ in $(V-K)_{0}$, a displacement similar to that exhibited in NGC 2506 by stars 2122 and 2212 (see Fig. 7), two definite proper motion and radial velocity members of that cluster. Note, however, that this separation is about three times the observed GB/AGB color difference in the globular cluster 47 Tucanae at a similar absolute magnitude (FPC81).

A few other stars in NGC 2204 deserve comment. The relatively low CO value of star 1129 [see Fig. 12(a)] is consistent with Dawson's (1981) claim (based upon DDO photometry) that it is metal poor; the star lies on the cluster GB in Fig. 3 but is marginally displaced from the field giant line in Fig. 10(a). The luminosity filter of Geisler's (1987) Washington photometry and Dawson's DDO colors indicate that star $\mathbf{4 2 1 0}$ is a field star, but no evidence suggests this here. Although no CO measurement was made for 4210 , its colors are consistent with cluster membership [see Fig. 9 (a) ]; it appears to be a clump star. Stars 3215 and 4223 may also be clump stars. Finally, star 1136 is reported to be variable by both Hawarden (1976a) and Dawson (1981); the infrared photometry for this star is given in Table 4(b). The dispersion in the measurements is consistent with the observational uncertainties alone, and the same is true of the infrared data for star 4132 , the brightest cluster member ob- served [see Table 4(c)]. However, if the NGC 2204 GB shows a curvature near its tip similar to that seen in the GB of 47 Tuc, then star 4132 is too blue to lie on the GB in Fig. 3.

\subsection{NGC 2243}

Table 5 presents the infrared photometry for the giants in NGC 2243. As may be seen in Fig. 4, except for star 1219, the few stars observed in NGC 2243 appear to define a narrow giant sequence in the infrared CMD. However, the optical data (Hawarden 1975; van den Bergh 1977; Bonifazi et al. 1990) suggest that stars 2308 and 4303 are clump stars. Star 1219 lies well to the blue of the GB in Fig. 4 and the optical CMDs and is most likely a field star. Unlike the other NGC 2243 giants, it lies near the field giant line in the ( $U$ $V)_{0},(V-K)_{0}$ diagram [Fig. 9(b) ], but it has a CO strength typical of the cluster in Fig. 12(a). Spectra of stars 4301 and 4303 by Friel (1989) indicate that 4303 is a radial velocity member but exclude 4301 from cluster membership. Because the radial velocities she measures for these two stars are very similar, we believe that 4301 is excluded on the basis of unusual line strengths, as Washington photometry (Geisler 1987) also assigns an extreme metallicity to this star; unfortunately, no CO index was measured for it here. We note, however, that the position of 4301 in the optical CMDs is unusual, lying below the clump but to the blue of the GB. It also deviates somewhat from the cluster norm in Fig. 9(b) and may well be a binary cluster member.

In reality, we may have observed only two true GB members of NGC 2243, stars 4110 and 4209. Due to a lack of evidence to the contrary, and with the assumption (see Sec. 6) that the GBs of the open clusters should lie nearly parallel to those of the globular clusters, we conclude that the random errors in the photometry of 4110 and 4209 cause these stars to be equally offset to the blue and red of the true GB position, respectively. This is consistent with stars 2308 and 4303 being clump stars and lying a bit to the blue of the GB (cf. the CMD of M67 in Fig. 2).

\subsection{NGC 2420}

Sarma \& Walker (1962), West (1967), Cannon \& Lloyd (1970), McClure et al. (1974), McClure et al. (1978), and

TABLE 5. NGC 2243 photometry.

\begin{tabular}{|c|c|c|c|c|c|c|c|c|c|c|c|c|c|}
\hline \multirow{2}{*}{ Stara } & \multicolumn{3}{|c|}{ Observed ${ }^{b}$} & \multicolumn{8}{|c|}{ Reddening Corrected $^{c}$} & \multirow{2}{*}{$\mathrm{n}$} & \multirow{2}{*}{ Notes } \\
\hline & $K$ & $J-K$ & $H-K$ & $K_{\mathrm{o}}$ & $(U-V)_{0}$ & $(B-V)$ & $(V-K)$ & $(J-K)_{0}$ & $(H-K)_{0}$ & $\mathrm{H}_{2} \mathrm{O}$ & $\mathrm{CO}$ & & \\
\hline 4110 & $10.20(3)$ & $0.69(3)$ & 0.11 & 10.19 & 1.84 & 1.07 & 2.54 & 0.66 & 0.10 & $\ldots$ & 0.075 & 1 & 1 \\
\hline 1219 & 9.32 & 0.63 & 0.12 & 9.31 & 2.00 & 1.06 & 2.35 & 0.60 & 0.11 & $\ldots$ & 0.065 & 1 & 1 \\
\hline 4209 & 8.70 & 0.86 & 0.14 & 8.69 & 2.74 & 1.35 & 3.19 & 0.83 & 0.13 & 0.045 & 0.14 & 1 & 1 \\
\hline 2308 & $11.32(3)$ & $0.58(3)$ & 0.11 & 11.31 & 1.44 & 0.94 & 2.17 & 0.55 & 0.10 & $\ldots$ & $\ldots$ & 1 & 1 \\
\hline 4301 & $12.19(3)$ & $0.50(4)$ & $0.09(4)$ & 12.18 & 1.31 & 0.80 & 1.86 & 0.47 & 0.08 & $\ldots$ & $\ldots$ & 1 & 2 \\
\hline 4303 & $11.42(3)$ & $0.59(4)$ & $0.11(3)$ & 11.41 & 1.35 & 0.87 & 2.17 & 0.56 & 0.10 & $\ldots$ & $\ldots$ & 1 & 2 \\
\hline
\end{tabular}

aStar designations are from Hawarden (1975).

b Observational uncertainties in the infrared magnitudes and colors are \pm 0.02 mag unless otherwise noted (in units of hundredths of a magnitude in parentheses).

'Reddening ratios are those of a K0 star as given by Cohen et al. (1981).

Notes: (1) Photoelectric $U B V$ photometry from Table 2 of van den Bergh (1977) and CCD BV photometry from Bonifazi et al. (1990). (2) Photoelectric UBV photometry from Table 2 of van den Bergh (1977). 


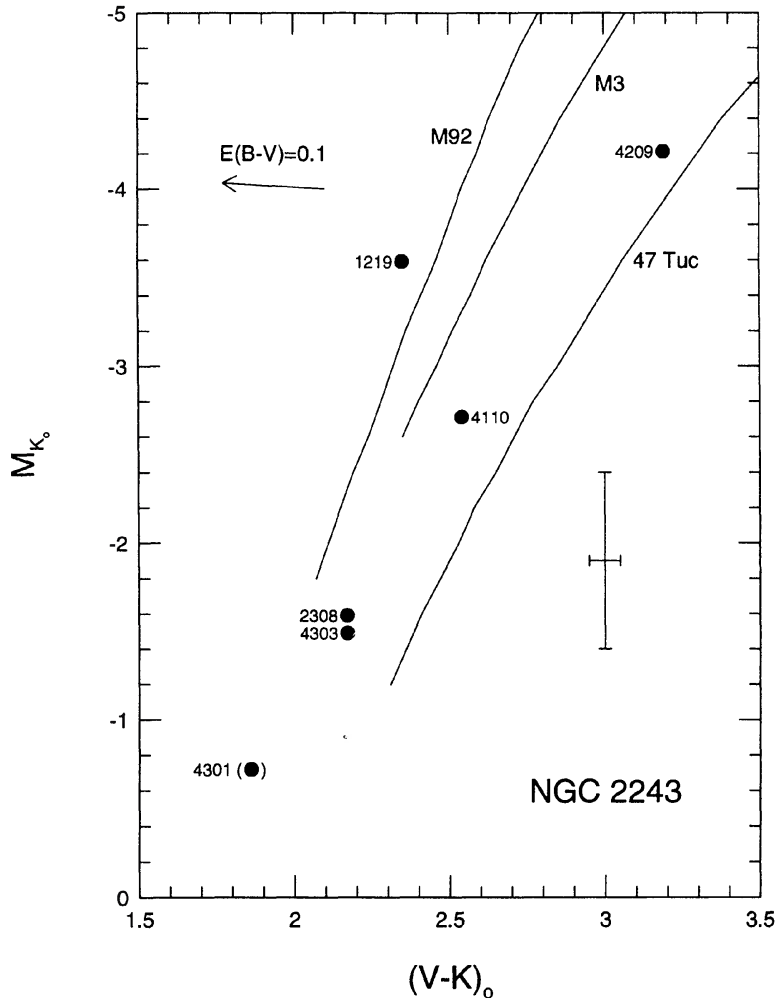

FIG. 4. Infrared CMD of NGC 2243. See the caption of Fig. 1 for further details.

Anthony-Twarog et al. (1990) have presented optical CMDs of NGC 2420. Our infrared data for this cluster are given in Table 6. The infrared CMD, shown in Fig. 5, is sparse.

Many radial velocity measurements have been made for NGC 2420 giants. McClure et al. (1974) found similar radi-

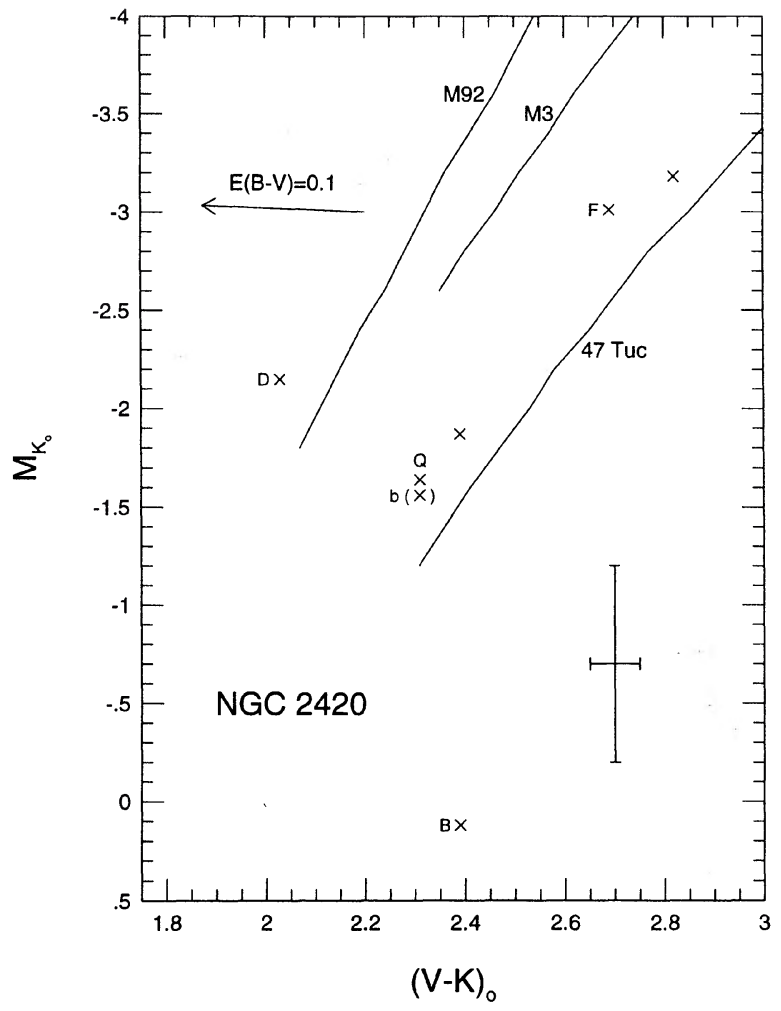

FIG. 5. Infrared CMD of NGC 2420. See the caption of Fig. 1 for further details.

al velocities for stars A, D, and X. Cameron \& Reid (1987) observed stars B and II-3-44; Liu \& Janes (1987) studied stars A, D, F, Q, U, and X; Smith \& Suntzeff (1987) looked at stars A, D, F, and X; Friel (1989) examined stars A, F, b, and II-3-44; and Friel et al. (1989) observed stars D and Q. From this list, only star $b$ has been classified as a radial veloc-

TABLE 6. NGC 2420 photometry.

\begin{tabular}{|c|c|c|c|c|c|c|c|c|c|c|c|c|c|}
\hline \multirow{2}{*}{ Star $^{a}$} & \multicolumn{3}{|c|}{ Observed $^{b}$} & \multicolumn{8}{|c|}{ Reddening Corrected ${ }^{c}$} & \multirow{2}{*}{$\mathrm{n}$} & \multirow{2}{*}{ Notes } \\
\hline & $K$ & $J-K$ & $H-K$ & $K_{\mathrm{o}}$ & $(U-V)_{\mathrm{o}}$ & $(B-V)_{C}$ & $V-K)_{0}$ & $(J-K)_{0}$ & $H-K)_{0}$ & $\mathrm{H}_{2} \mathrm{O}$ & $\mathrm{CO}$ & & \\
\hline A & 8.63 & $0.70(3)$ & 0.10 & 8.62 & 2.45 & 1.24 & 2.82 & 0.69 & 0.10 & 0.04 & 0.095 & 1 & 1 \\
\hline B & $11.93(5)$ & $0.67(6)$ & $0.03(5)$ & 11.92 & 1.24 & 0.83 & 2.39 & 0.66 & 0.03 & $\ldots$ & $\ldots$ & 1 & 1 \\
\hline D & 9.66 & $0.48(3)$ & 0.08 & 9.65 & 1.41 & 0.88 & 2.03 & 0.47 & 0.08 & 0.00 & -0.02 & 1 & 1 \\
\hline $\mathrm{F}$ & 8.80 & $0.69(3)$ & 0.11 & 8.79 & 1.92 & 1.11 & 2.69 & 0.68 & 0.11 & 0.01 & 0.07 & 1 & 2 \\
\hline Q & 10.17 & $0.56(3)$ & 0.09 & 10.16 & 1.66 & 0.97 & 2.31 & 0.55 & 0.09 & 0.01 & 0.03 & 1 & 1 \\
\hline U & 9.94 & $0.58(3)$ & 0.09 & 9.93 & 1.80 & 1.01 & 2.39 & 0.57 & 0.09 & $\ldots$ & 0.05 & 1 & 1 \\
\hline$x$ & 8.65 & $0.66(3)$ & 0.12 & 8.64 & 2.52 & 1.37 & 2.67 & 0.65 & 0.12 & -0.005 & 0.09 & 1 & 2,4 \\
\hline b & 10.25 & $0.55(3)$ & 0.09 & 10.24 & 1.70 & 0.98 & 2.31 & 0.54 & 0.09 & $\ldots$ & 0.07 & 1 & 1 \\
\hline II-3-44 & 11.19 & $0.53(4)$ & 0.09 & 11.18 & $\ldots$ & 0.92 & 2.22 & 0.52 & 0.09 & $\ldots$ & $\ldots$ & 1 & 3,4 \\
\hline
\end{tabular}

aStar designations are from West (1967).

b Observational uncertainties in the infrared magnitudes and colors are \pm 0.02 mag unless otherwise noted (in units of hundredths of a magnitude in parentheses).

CReddening ratios are those of a K0 star as given by Cohen et al. (1981).

Notes: (1) Photoelectric UBV photometry from the adopted sequence of McClure et al. (1974) and CCD BV photometry from Anthony-Twarog et al. (1990). (2) Photoelectric UBV photometry from the adopted sequence of McClure et al. (1974). (3) CCD BV photometry from Anthony-Twarog et al. (1990). (4) Proper motion non-member (van Altena \& Jones 1970); not included in figures. 
ity nonmember of NGC 2420, but stars X and II-3-44 are excluded by proper motion surveys (see Sec. 2.5 ).

Among the NGC 2420 giants to be discussed individually, star $B$ is especially intriguing. It lies at the base of the GB in optical CMDs but is well to the red of the GB in Fig. 5 and deviates significantly from the cluster trends in Figs. 9(b), $10(\mathrm{~b})$, and 11(b). Although the uncertainties in its infrared photometry are somewhat larger than the norms, this cannot explain its discrepant position in these figures. While all of the other NGC 2420 giants lie below the globular cluster/ field giant region of Fig. 10(b), star B lies above it. Any correction applied to this cluster's data to remove its systematic deviation in this diagram (see Sec. 5.2) would cause star $B$ to become even more discrepant. Thus, it may be variable, it may be a field star, or its photometry may be seriously in error. As mentioned in Sec. 2.5, it is not included in the proper motion study of van Altena \& Jones (1970); however, it meets Cannon \& Lloyd's (1970) and Cabrera-Caño \& Alfaro's (1990) criteria for probable membership, and it is classified as a radial velocity member by Cameron \& Reid (1987). Star B was not measured in the CO band here, so no other information is available to clarify its nature.

Stars b and Q may be clump stars. Friel (1989) finds star b to be a radial velocity nonmember, and its does have a high $\mathrm{CO}$ index with respect to the cluster trend in Fig. 12(c). Star $\mathrm{D}$, which lies considerably blueward of the GB, is a known mild Ba II star and binary (Liu \& Janes 1987; Smith \& Suntzeff 1987). The questionable proper motion membership of star $F$ is described in Sec. 2.5. It lies to the blue of each $B V$ giant branch mentioned above and may be an AGB star. $F$ is described as a background supergiant by Sowell (1987) and deviates from the trend exhibited by the other cluster stars in Fig. 9(b), but its $\mathrm{CO}$ strength is not anomalous.

\section{6 NGC 2477}

The infrared data for NGC 2477 are in Table 7, while its CMD is given in Fig. 6. In spite of the variable reddening of the cluster, the infrared CMD shows surprisingly little scatter. In contrast, the optical CMD presented by HHM is influenced not only by the variable reddening but also by large random and systematic errors in the red giant $B V$ photographic photometry. The photoelectric $V$ magnitudes for cluster giants in Tables 1 and 2 of HHM are all fainter (an average of $0.08 \mathrm{mag}$ ) than the photographic values of their Tables 1 and 3 for the ten stars in common between these three tables. Mermilliod \& Claria (1990) have collected photoelectric $U B V$ photometry and Mermilliod (1990) has measured radial velocities for all of the stars in Fig. 6 except 4035; the photometry supports the photoelectric values of HHM, and the radial velocities indicate that all of these stars are cluster members, with star 1044 being a long period ( 12 $15 \mathrm{yr}$ ) binary.

Having hopefully accounted for the photometric uncertainties and variable reddening of this cluster, we can address the positions of the observed giants in Fig. 6 more confidently. We do not seem to have observed any of the clump stars in NGC 2477; star 8019 lies at a similar luminosity but to the red of the clump in the optical CMD of HHM. Star 6053 lies blueward of the GB in the optical CMD, but this is probably due to the previously mentioned photometric errors; its location in Figs. 6 and 12(c) is consistent with membership. Star 4035 lies about $1 \mathrm{mag}$ to the red of the GB in Fig. 6, contrary to the claim of HHM that it is a GB star. It may be a nonmember (Bessell et al. 1983, hereafter referred

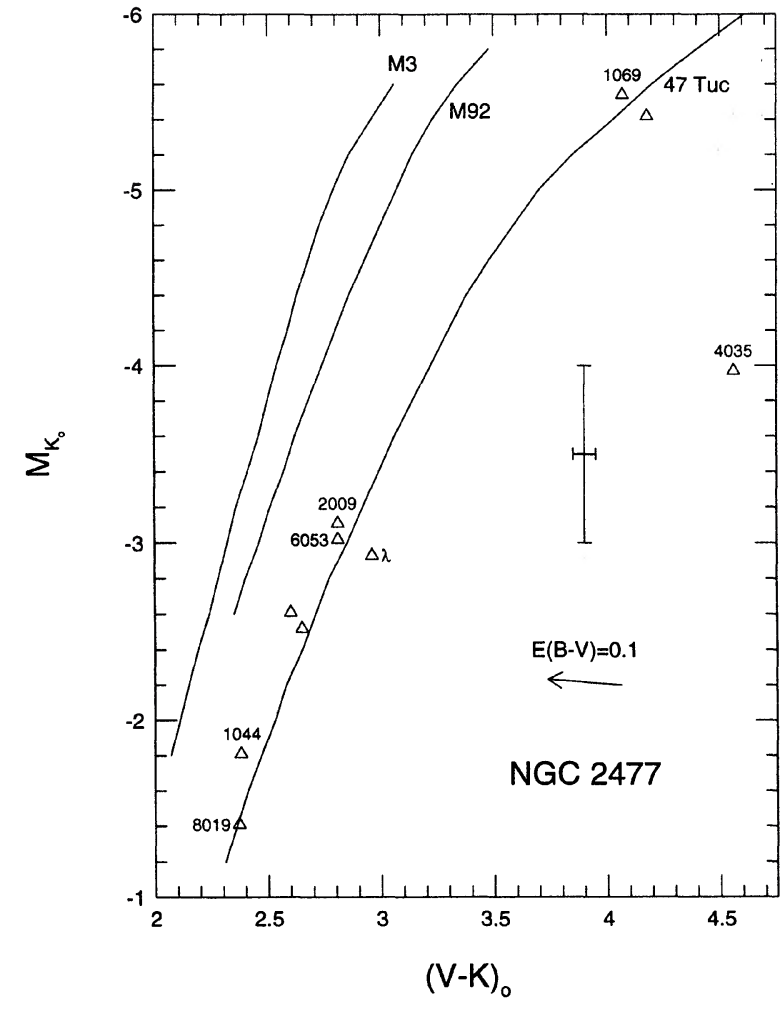

FIG. 6. Infrared CMD of NGC 2477. Stars C2 and 1220, carbon stars, are not shown. C2 lies on a red extension of the GB at $M_{K}=-7.25,(V-$ $K)_{0}=6.50 ; 1220$ lies far to the red of the GB at $M_{K}=-4.89$, ( $V$ $K)_{0}=6.68$ and is a probable nonmember. See the caption of Fig. 1 for further details.

to as BWLE) or a variable. Its photographic $V$ magnitude (HHM) differs by 0.19 mag from the photoelectric value of Eggen (1974), a difference considerably greater than the aforementioned systematic error in HHM's data but similar to the difference between the photographic HHM photometry and Mermilliod's (1990) photoelectric data for stars 2009 and 6053. Stars C2 and 1220 (not shown in Fig. 6) are carbon stars and variables (Catchpole \& Feast 1973; Eggen 1974; Hartwick \& Hesser 1974; BWLE); they will be discussed in Sec. 4.9.

Star $\lambda$ is red of both the optical and infrared GBs; it could be a field star, but its CO strength [see Fig. 12(c)] agrees with other cluster members of its color. However, $\lambda$ (along with star 8019) may instead represent the cluster GB, with all of the other cluster giants observed here being AGB stars. The $H-R$ diagram for this cluster [Fig. 14(b)] could be interpreted as that of a cluster which has not yet undergone the red giant branch (RGB) phase transition (Renzini \& Buzzoni 1986); the difficulty encountered by Barbaro \& Pigatto (1984) in reproducing the GB luminosity function of NGC 2477 and other open clusters of similar age leads them to the same conclusion. Although the mass we use to compute the stellar parameters for this cluster in Sec. 6 is less than the transition mass between degenerate and nondegenerate helium ignition, $M_{\mathrm{HeF}}$, in classical models of stellar evolution (e.g., $M_{\mathrm{HeF}} \sim 2.2 M_{\odot}$ for $Y=0.30$ and $Z=0.02$ according to Sweigart et al. 1990), it is greater than that predicted by models which include even a modest amount of 
TABLE 7. NGC 2477 photometry.

\begin{tabular}{|c|c|c|c|c|c|c|c|c|c|c|c|c|c|c|}
\hline \multirow{2}{*}{ Star ${ }^{a}$} & \multirow{2}{*}{$\mathrm{E}(B-V)^{\mathrm{b}}$} & \multicolumn{3}{|c|}{ Observedc } & \multicolumn{8}{|c|}{ Reddening Corrected ${ }^{\mathrm{d}}$} & \multirow{2}{*}{$\mathrm{n}$} & \multirow{2}{*}{ Notes } \\
\hline & & $K$ & $J-K$ & $H-K$ & $K_{\mathrm{o}}$ & $(U-V)_{0}($ & $B-V)_{0}$ & $(V-K)_{0}$ & $(J-K)_{0}$ & $(H-K)_{0}$ & $\mathrm{H}_{2} \mathrm{O}$ & $\mathrm{CO}$ & & \\
\hline 1044 & 0.21 & 8.76 & 0.70 & 0.15 & 8.69 & 1.99 & 1.08 & 2.38 & 0.56 & 0.10 & $\ldots$ & 0.07 & 1 & 1 \\
\hline 1069 & 0.22 & 5.03 & 1.09 & 0.24 & 4.96 & 3.73 & 1.66 & 4.07 & 0.95 & 0.19 & $0.065(3)$ & 0.20 & 2 & 2,9 \\
\hline 2009 & 0.22 & 7.46 & 0.81 & 0.17 & 7.39 & 2.59 & 1.27 & 2.81 & 0.67 & 0.12 & $\ldots$ & 0.105 & 1 & 3 \\
\hline 4035 & 0.22 & 6.60 & 1.19 & 0.25 & 6.53 & 3.88 & 1.73 & 4.56 & 1.05 & 0.20 & $0.075(3)$ & 0.18 & 1 & 4,9 \\
\hline 4067 & 0.22 & 8.05 & 0.77 & 0.14 & 7.98 & 2.39 & 1.20 & 2.65 & 0.63 & 0.09 & $\ldots$ & 0.085 & 1 & 2 \\
\hline 6053 & 0.18 & 7.54 & 0.80 & 0.16 & 7.48 & 2.61 & 1.26 & 2.81 & 0.68 & 0.12 & $\ldots$ & 0.095 & 2 & 3 \\
\hline 8019 & 0.18 & 9.15 & 0.67 & 0.14 & 9.09 & 1.98 & 1.09 & 2.37 & 0.55 & 0.10 & $\ldots$ & 0.065 & 1 & 2 \\
\hline 2117 & 0.22 & 5.15 & 1.10 & 0.25 & 5.08 & 3.74 & 1.69 & 4.18 & 0.96 & 0.20 & $0.055(3)$ & 0.19 & 1 & 3,10 \\
\hline$\theta$ & 0.28 & 7.98 & 0.80 & 0.17 & 7.89 & 2.31 & 1.17 & 2.60 & 0.62 & 0.10 & ... & 0.10 & 1 & 2 \\
\hline$\lambda$ & 0.25 & 7.65 & 0.87 & 0.19 & 7.57 & 2.77 & 1.32 & 2.96 & 0.71 & 0.13 & ... & 0.10 & 1 & 2 \\
\hline 1220 & 0.22 & 5.69 & 1.84 & 0.64 & 5.61 & ... & 3.20 & 6.68 & 1.69 & 0.58 & $0.215(3)$ & 0.01 & 1 & 5,7 \\
\hline $\mathrm{C} 2$ & 0.22 & 3.33 & 1.68 & 0.54 & 3.25 & ... & 2.79 & 6.50 & 1.53 & 0.48 & $0.085(3)$ & 0.21 & 1 & 6,8 \\
\hline
\end{tabular}

aStar designations are from Hartwick et al. (1972).

bFor the seven stars with $(V-K)_{0}$ less than $3.00 \mathrm{mag}$, the reddenings are determined by assuming that the unreddened colors coincide with the field giant relation in the $(U-V)_{0},(V-K)_{0}$ diagram (Fig. 9b). The average reddening of these seven stars is assumed for the giants with redder $(V-K)_{0}$ colors.

cObservational uncertainties in the infrared magnitudes and colors are \pm 0.02 mag unless otherwise noted (in units of hundredths of a magnitude in parentheses).

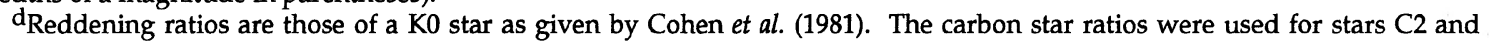
1220.

Notes: (1) Photoelectric UBV photometry from Hartwick et al. (1972) and Mermilliod \& Claria (1990). (2) Photoelectric UBV photometry from Hartwick et al. (1972), Eggen (1974) and Mermilliod \& Claria (1990). (3) Photoelectric UBV photometry from Mermilliod \& Claria (1990). (4) Photoelectric UBV photometry from Eggen (1974). (5) Photoelectric BV photometry from Hartwick \& Hesser (1974). (6) Photoelectric $B V$ photometry from Hartwick \& Hesser (1974) and Eggen (1974). (7) Carbon star and variable; range in $V$ is $\sim 0.2$ mag. $(K-L)_{0}=0.56$. Not included in figures. (8) Same as note 7, but range in $V$ is $\sim 0.4 \mathrm{mag}$, and $(K-L)_{0}=0.34$. (9) $(K-L)_{\mathrm{O}}=0.12$. (10) $(K-L)_{\mathrm{o}}=0.14$.

convective overshooting (e.g., Maeder \& Meynet 1989 give $M_{\mathrm{HeF}}=1.85 M_{\odot}$ for $Y=0.28$ and $Z=0.02$ ). Nonetheless, we assume here that the classical limits apply and that the displacement of star $\lambda$ from the cluster trend in the CMD is due to other factors.

\subsection{NGC 2506}

Table 8 contains the infrared data for NGC 2506, while the corresponding CMD is shown in Fig. 7. The optical CMD consulted for NGC 2506 is that of McClure et al. (1981). The appearance of both the optical and infrared CMDs suggests that stars 2401 and 2402 are GB stars, 2122 and 2212 are AGB stars (proper motion membership probability $\geqslant 90 \%$, see Sec. 2.5 ), 1112, 4205, and 4240 are clump stars, and 4402 is either a nonmember or highly variable. Radial velocity measurements of stars $1112,2122,2212$, 3254,4205 , and 4240 by Mathieu \& Latham (1990) indicate that all of theses stars are cluster members, although we consider 3254 to be a field star due to its proper motion (see Sec. 2.5). Friel (1989) also classifies star 2212 as a radial velocity member of the cluster. Although only two radial velocity measurements were made per star, the possibility that stars 2122 and 2212 are short-period photometric binaries (rather than AGB stars) appears to be ruled out by the Mathieu \& Latham data. Proper motion or radial velocity data for stars 2401 and 2402 would allow us to define the GB location of NGC 2506 with greater confidence.

\subsection{NGC 2660}

Table 9 lists the infrared data for NGC 2660, and Fig. 8 illustrates its CMD. All of the stars observed in NGC 2660 are brighter than the highly populated clump seen in the optical CMD of Hartwick \& Hesser (1973); the optical GB has a similar appearance to Fig. 8. Star 9009, with ( $V$ $K)_{0}=6.58$, is too red to have been included in Fig. 8 but lies on a reasonable extension of the GB. It is a carbon star (Hartwick \& Hesser 1971; BWLE), is variable (Eggen 1972b; Hartwick \& Hesser 1973) and will be discussed in Sec. 4.9 with the carbon stars of NGC 2477.

The scatter present in Fig. 8 prevents us from determining an accurate locus for the GB of NGC 2660, but the following observations lead us to believe that the best GB candidates are stars 9016 and 4304 . Star 4224 may be variable, as suggested by the $B V$ photometry of Hartwick \& Hesser (1973) and the infrared photometry of BWLE (their $K$ magnitude for the star is 0.07 mag fainter than ours). ${ }^{3}$ This could explain why 4224 lies to the blue of the apparent GB in the infrared, is the only cluster member lying on the field relation in Fig. 10(b), and has an anomalously high DDO metallicity index (Hesser \& Smith 1987). Since stars 4224 and 4304 are the only two noncarbon stars in NGC 2660 with $\mathrm{CO}$ measurements [see Fig. 12(d)], it is not clear whether

\footnotetext{
${ }^{3}$ The infrared photometry of BWLE has been transformed to the CTIO
} system using the relations in Table II of Elias et al. (1983). 
TABLE 8. NGC 2506 photometry.

\begin{tabular}{|c|c|c|c|c|c|c|c|c|c|c|c|c|c|}
\hline \multirow{2}{*}{ Stara } & \multicolumn{3}{|c|}{ Observed ${ }^{b}$} & \multicolumn{8}{|c|}{ Reddening Corrected ${ }^{c}$} & \multirow{2}{*}{$\mathrm{n}$} & \multirow{2}{*}{ Notes } \\
\hline & $K$ & $J-K$ & $H-K$ & $K_{o}$ & $(U-V)$ & $B-V)$ & $V-K)_{C}$ & $(J-K)_{0}$ & $H-K)_{0}$ & $\mathrm{H}_{2} \mathrm{O}$ & $\mathrm{CO}$ & & \\
\hline 1112 & 10.64 & $0.60(3)$ & $0.10(3)$ & 10.62 & 1.49 & 0.93 & 2.15 & 0.57 & 0.09 & $\ldots$ & $\ldots$ & 1 & 1 \\
\hline 2122 & 9.04 & 0.69 & 0.12 & 9.02 & 2.02 & 1.08 & 2.53 & 0.66 & 0.11 & $\ldots$ & 0.10 & 1 & 1 \\
\hline 2212 & 9.39 & 0.65 & 0.11 & 9.37 & 1.81 & 1.02 & 2.40 & 0.62 & 0.10 & $\ldots$ & 0.085 & 1 & 1 \\
\hline 3254 & 7.71 & 0.87 & 0.16 & 7.69 & 2.80 & 1.36 & 3.26 & 0.84 & 0.15 & 0.035 & 0.125 & 1 & 1,3 \\
\hline 4205 & 10.93 & $0.58(3)$ & 0.10 & 10.91 & $\ldots$ & 0.90 & 2.20 & 0.55 & 0.09 & $\ldots$ & $\ldots$ & 1 & 2 \\
\hline 4228 & 7.77 & 1.02 & 0.20 & 7.75 & 3.29 & 1.61 & 4.07 & 0.99 & 0.19 & 0.065 & 0.19 & 2 & 1,4 \\
\hline 4240 & 10.71 & 0.62 & 0.11 & 10.69 & 1.59 & 0.94 & 2.26 & 0.59 & 0.10 & $\ldots$ & $\ldots$ & 1 & 1 \\
\hline 2401 & 7.28 & 0.94 & 0.18 & 7.26 & 3.44 & 1.54 & 3.65 & 0.91 & 0.17 & 0.05 & 0.155 & 1 & 1 \\
\hline 2402 & 9.60 & 0.71 & 0.12 & 9.58 & 2.17 & 1.14 & 2.69 & 0.68 & 0.11 & $\ldots$ & $\ldots$ & 1 & 1 \\
\hline 4402 & 7.98 & 1.06 & 0.22 & 7.96 & 3.72 & 1.68 & 4.44 & 1.03 & 0.21 & $0.07 \quad(3)$ & 0.18 & 1 & 1 \\
\hline
\end{tabular}

aStar designations are from McClure et al. (1981).

b Observational uncertainties in the infrared magnitudes and colors are \pm 0.02 mag unless otherwise noted (in units of hundredths of a magnitude in parentheses).

CReddening ratios are those of a K0 star as given by Cohen et al. (1981).

Notes: (1) Photoelectric UBV photometry from McClure et al. (1981). (2) Photographic BV photometry from McClure et al. (1981). (3) Proper motion non-member (Chiu \& van Altena 1981); not included in figures. (4) Same as note 3, plus $(K-L)_{0}=0.16$.

either is representative of the cluster in general. Although Hesser \& Smith derive extreme reddenings for 4224 and $4304[\mathrm{E}(B-V)=0.44$ and 0.52 , respectively], Mermilliod's (1990) radial velocity measurements indicate that both stars are cluster members. Mermilliod did not observe star 9016, but he classifies stars 2121, 9023, and 9025 as

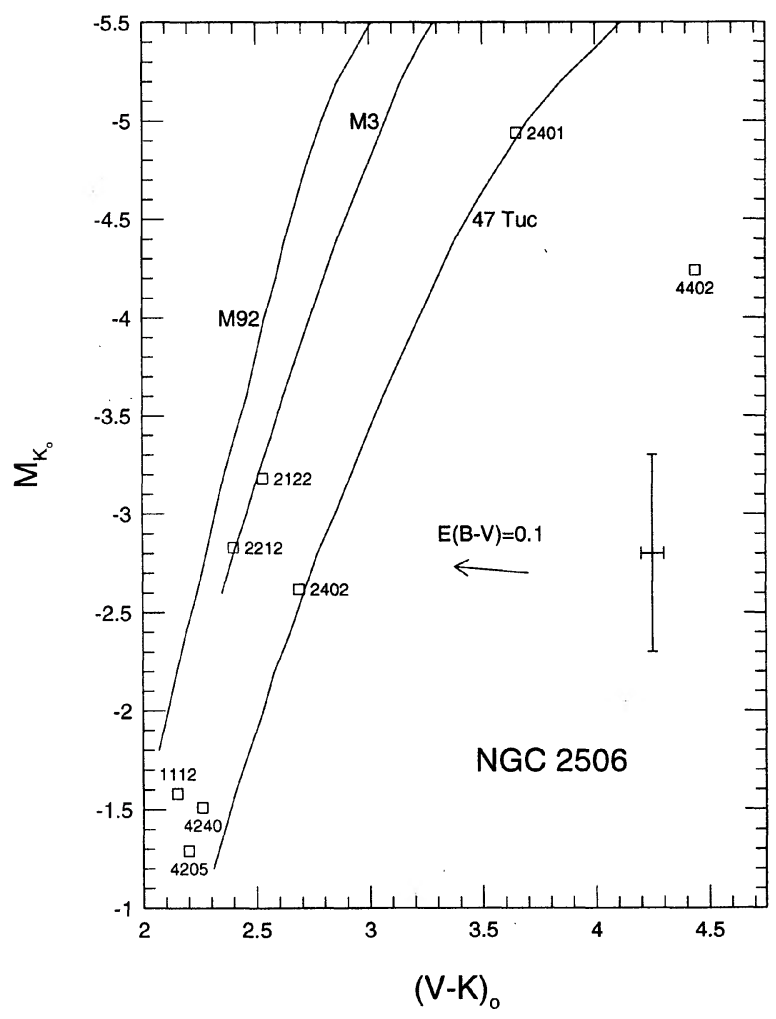

FIG. 7. Infrared CMD of NGC 2506. See the caption of Fig. 1 for further details. radial velocity nonmembers of NGC 2660; the first two of these may be variable as well. There is a 0.08 mag difference between our and BWLE's $J-K$ color for 2121. While our data agree with that of BWLE for 9023, Hesser \& Smith's DDO photometry suggests that it is a possible variable; their colors also support star 9025 being a nonmember. Note that both 9023 and 9025 lie to the blue of the field giant relation in Fig. 9(b); star 9016 does not.

\subsection{The Carbon Stars}

The three carbon stars we observed - stars C2 and 1220 in NGC 2477, and star 9009 in NGC 2660 - have $J H K$ colors similar to other galactic carbon stars (Cohen et al. 1981). The $\mathrm{CO}$ and $\mathrm{H}_{2} \mathrm{O}$ indices of 1220 and 9009 also agree with their field counterparts, while $\mathrm{C} 2$ seems to have low $\mathrm{CO}$ and high $\mathrm{H}_{2} \mathrm{O}$ for its $(J-K)_{0}$ color.

The cluster membership of the three $\mathrm{C}$ stars can be explored by comparing their bolometric luminosities (derived in Sec. 6 and listed in Table 10) to Magellanic Cloud (MC) C star luminosity functions presented in Fig. 9 of Cohen et al. (1981) and Figs. 12 and 13 of Frogel et al. (1990). Stars C2 and 9009 have bolometric luminosities which would put them at the faint and bright ends of the $\mathrm{MC}$ distributions, respectively. Star C2, with $M_{\text {bol }}=-4.08$, may have been observed in a subluminous post-thermal pulse phase, which could also explain its observed molecular band indices. Its cluster membership is supported by the fact that its radial velocity is similar to that of star 1069 in NGC 2477 (Catchpole \& Feast 1973). Star $9009\left(M_{\text {bol }}=-5.34\right)$ is slightly brighter than the C stars in SWB V clusters (Frogel et al. 1990), which have an age similar to that of NGC 2660; however, this is expected from the metallicity difference between the average SWB V cluster and NGC 2660. Star 1220, on the other hand, with $M_{\text {bol }}=-1.68$, appears to be a reddened background star, as its position in Fig. 6, the optical photometry of Hartwick \& Hesser (1974), and the infrared work of BWLE also imply. Overall, proper motion or radial velocity measurements would permit the cluster membership of all three of these $\mathrm{C}$ stars to be put on a firmer footing. 
TABLE 9. NGC 2660 photometry.

\begin{tabular}{|c|c|c|c|c|c|c|c|c|c|c|c|c|c|}
\hline \multirow{2}{*}{ Stara } & \multicolumn{3}{|c|}{ Observed $^{b}$} & \multicolumn{8}{|c|}{ Reddening Correctedc } & \multirow{2}{*}{$\mathrm{n}$} & \multirow{2}{*}{ Notes } \\
\hline & K & $J-K$ & $H-K$ & $K_{\mathrm{o}}$ & $(U-V)_{\mathrm{o}}$ & $(B-V)$ & $(V-K)_{\mathrm{c}}$ & $(J-K)_{0}$ & $(H-K)_{0}$ & $\mathrm{H}_{2} \mathrm{O}$ & $\mathrm{CO}$ & & \\
\hline 9009 & 3.88 & $1.98(3)$ & 0.73 & 3.74 & $\ldots$ & 3.86 & 6.58 & 1.71 & 0.63 & $0.185(7)$ & 0.035 & 2 & 3 \\
\hline 9016 & 10.55 & $0.85(4)$ & 0.17 & 10.42 & 1.72 & 0.98 & 2.19 & 0.60 & 0.08 & ... & $\ldots$ & 1 & 1 \\
\hline 9023 & 10.12 & $0.84(4)$ & 0.16 & 9.99 & 1.82 & 0.99 & 2.12 & 0.59 & 0.07 & $\ldots$ & $\cdots$ & 1 & 1 \\
\hline 9025 & 9.37 & $0.89(4)$ & 0.18 & 9.24 & 2.29 & 1.10 & 2.37 & 0.64 & 0.09 & $\ldots$ & $\ldots$ & 1 & 1 \\
\hline 2121 & 9.73 & $0.84(4)$ & 0.16 & 9.60 & $\ldots$ & 0.93 & 2.02 & 0.59 & 0.07 & $\ldots$ & $\ldots$ & 1 & 2 \\
\hline 4224 & 8.48 & $0.82(3)$ & 0.18 & 8.35 & $\ldots$ & 1.44 & 2.27 & 0.57 & 0.09 & $0.045(7)$ & 0.145 & 2 & 2 \\
\hline 4304 & 7.59 & $1.11(4)$ & 0.22 & 7.46 & $\ldots$ & 1.48 & 3.00 & 0.86 & 0.13 & $0.045(7)$ & 0.175 & 1 & 4 \\
\hline
\end{tabular}

aStar designations are from Hartwick \& Hesser (1973).

b Observational uncertainties in the infrared magnitudes and colors are \pm 0.02 mag unless otherwise noted (in units of hundredths of a magnitude in parentheses).

'Reddening ratios are those of a K0 star as given by Cohen et al. (1981). The carbon star ratios were used for star 9009.

Notes: (1) Photoelectric $U B V$ photometry from Hartwick \& Hesser (1973). (2) Photoelectric $B V$ photometry from Hartwick \& Hesser (1973). (3) Photoelectric BV photometry from Hartwick \& Hesser (1973) and Eggen (1972b, 1974). Carbon star and variable; range in $V$ is $\sim 0.5$ mag. $(K-L)_{0}=0.54$. Not included in figures. (4) Photographic $B V$ photometry from Hartwick \& Hesser (1973).

\section{THE COLOR-COLOR DIAGRAMS \\ 5.1 The UVK Colors}

The $(U-V)_{0},(V-K)_{0}$ diagram for stars from M67 and NGC 2204 is presented in Fig. 9(a); Fig. 9(b) is a similar plot for the other six open clusters we observed. For ( $V_{-}$

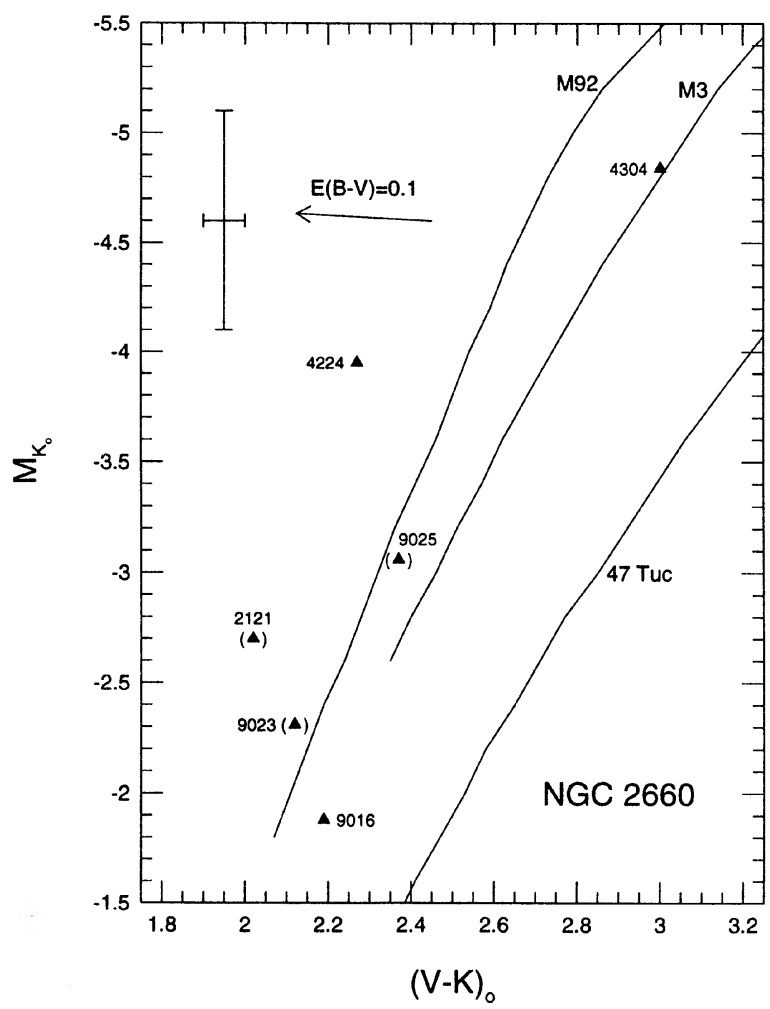

FIG. 8. Infrared CMD of NGC 2660. Star 9009 is not shown. It is a carbon star and lies on a red extension of the GB at $M_{K}=-8.56$, ( $V$ $K)_{0}=6.58$. See the caption of Fig. 1 for further details.
$K)_{0}<3.5$, the stars of each open cluster show a fairly linear dependence on $(U-V)_{0}$, and their locations are consistent with the metallicity related trend shown by globular cluster giants (FPC83). For example, stars from M67, with a nearsolar abundance, lie on the line defined by field giants. ${ }^{4}$ The open cluster giants that lie between the field giant line and the line defined by the metal-poor globular clusters (M3, M13, and M92) are primarily from the most metal-poor clusters listed in Table 1. The abundances of these clusters are comparable to or slightly greater than those of the most metal-rich globular clusters, whose giants occupy a similar intermediate position in the $(U-V)_{0},(V-K)_{0}$ plane (see Fig. 1 of FPC83).

The stars of a specific cluster which do not follow that cluster's general trend in Fig. 9 [and especially those to the blue of the field line in $\left.(V-K)_{0}\right]$ are all suspected field stars or have indications of chemical peculiarity (see Sec. 4). These include stars 2261 (and possibly 2239) in Melotte 66; F117, III-34, IV-20, T574, and T654 in M67; 1329 in NGC 2204; 1219 and 4301 in NGC 2243; B and F in NGC 2420; and 9023 and 9025 in NGC 2660. Since errors in $V$ will move stars along tracks nearly parallel to the field giant line over most of the region of interest, they can be effectively ignored as a source of any disagreement. We also discount problems with the $K$ photometry because of the close agreement between observations from CTIO and Las Campanas. Therefore, deviations of any star from the norm for its cluster are most likely due to nonuniform reddening, photometric errors in $U$, or nonmembership. All of the proper motion nonmembers for which $U-V$ colors are available (not shown in Fig. 9) deviate from the linear trend of the respective cluster

${ }^{4}$ If the literature average of $\mathrm{E}(B-V)=0.05$ is used for M67, its giants will lie systematically to the blue in $(V-K)_{0}$ of the mean line for field giants in Fig. 9(a). It is unlikely that this could be caused by errors in the $U-V$ photometry. The photoelectric $U-V$ colors of Eggen \& Sandage (1964) for M67 agree with those of Sturch $(1972,1973)$, although the number of stars in common is small, and with the photographic values of Johnson \& Sandage (1955) and Racine (1971). A value of $\mathrm{E}(B-V)=0.03$, on the other hand, based on the recent work of Nissen et al. (1987) and Fig. 5 of Burstein et al. (1986) brings the cluster stars into good agreement with the field giant relation in Fig. 9(a). 


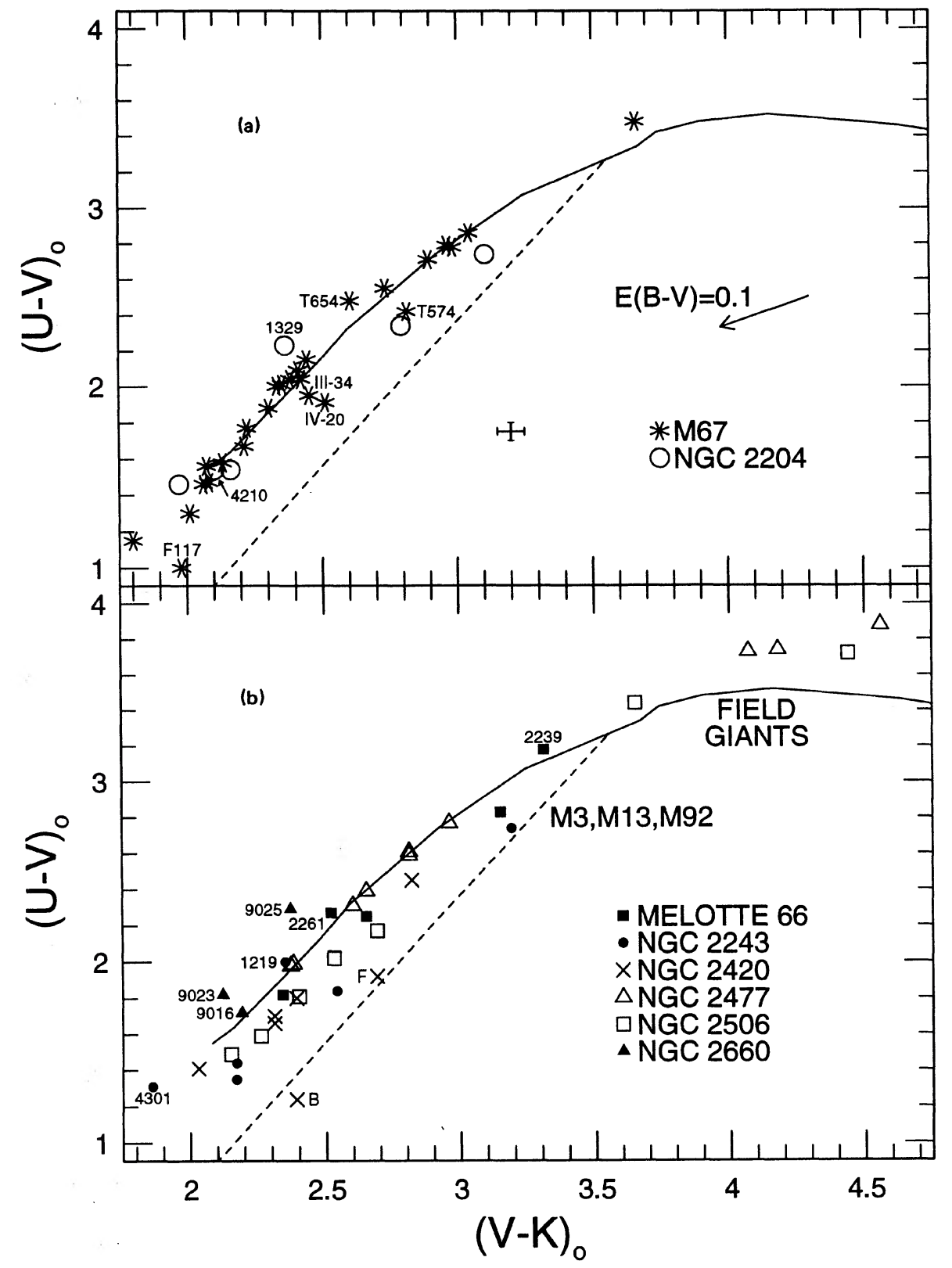

FIG. 9. $(U-V)_{0},(V-K)_{0}$ colors of the open cluster giants. Stars F94 and F115 in M67 are not shown. Interesting stars discussed in the text are individually labeled. The field relation is taken from Table 1 of Johnson (1966), amended by Table 3 of Lee (1970) for the M stars. The relation for the globular clusters M3, M13, and M92 is taken from Fig. 2(a) of CFP. The error bars represent the $\pm 1 \sigma$ uncertainties in the observed colors; the effect of increasing the value of $\mathrm{E}(B-V)$ by 0.1 mag is also shown. stars. $U$-band photometry for a greater proportion of the open cluster giants would allow a more complete analysis of the cluster trends seen in Fig. 9.

The $(V-K)_{0}$ colors of the stars we observed in NGC 2477 will move considerably to the blue of the field giant line in Fig. 9(b) if either the individual stellar reddenings of HHM or the average literature value of $\mathrm{E}(B-V)=0.27$ is used. Photometric errors seem to be an unlikely explanation for this phenomenon, since the data of HHM, Eggen (1974) and Mermilliod (1990) are in close agreement in $U-V$, while our $K$ magnitudes agree with those of BWLE. Thus, we have assumed that the literature average of $\mathrm{E}(B-V)$ is incor- rect. Because this cluster exhibits highly variable reddening, the giants with $(V-K)_{0}<3.00$ have been individually dereddened to achieve agreement with the field giant relation in Fig. 9(b); the resultant average reddening of 0.22 mag has been assumed for the other NGC 2477 giants, and it is in close agreement with Eggen's $\mathrm{E}(B-V)$ of 0.23 mag derived from observations of field stars along the line of sight to NGC 2477. We feel that the linear color trends exhibited by the other clusters in this diagram justifies our approach; it also greatly reduces the observed scatter in the cluster CMD (Fig. 6). However, note that we have assumed that $\langle[\mathrm{Fe} / \mathrm{H}]\rangle=0.0$ for the giants that define the field relation 
in Fig. 9; significant deviations from this value could mean that the reddenings we have derived for NGC 2477 and M67 are incorrect.

\subsection{The VJK Colors}

The relation between $(J-K)_{0}$ and $(V-K)_{0}$ for the cluster giants we observed is displayed in Fig. 10. Because the globular cluster and field giant lines define a relatively narrow region in this figure and the reddening line is nearly parallel to both relations, this diagram is not useful for examining the membership of known giants. Any giant star observed should, in principle, lie between or very near these relations.
We note that all of the stars excluded on the basis of their proper motions occupy the same region of Fig. 10 as the cluster members do (see below for a discussion of the NGC 2420 nonmembers). In fact, most of the cluster stars lie within $1 \sigma$ of the mean relations. Some clusters do show more scatter than might be expected; M67 is especially noteworthy in this respect, but its scatter can be attributed to binaries or other anomalous stars (see Sec. 4.2). The three clusters that appear to systematically deviate from the region defined by the globular cluster giants and field giants in Fig. 10(b) are NGC 2420 and NGC 2477 to the red and NGC 2660 to the blue in $(V-K)_{0}$. These will be discussed in turn.

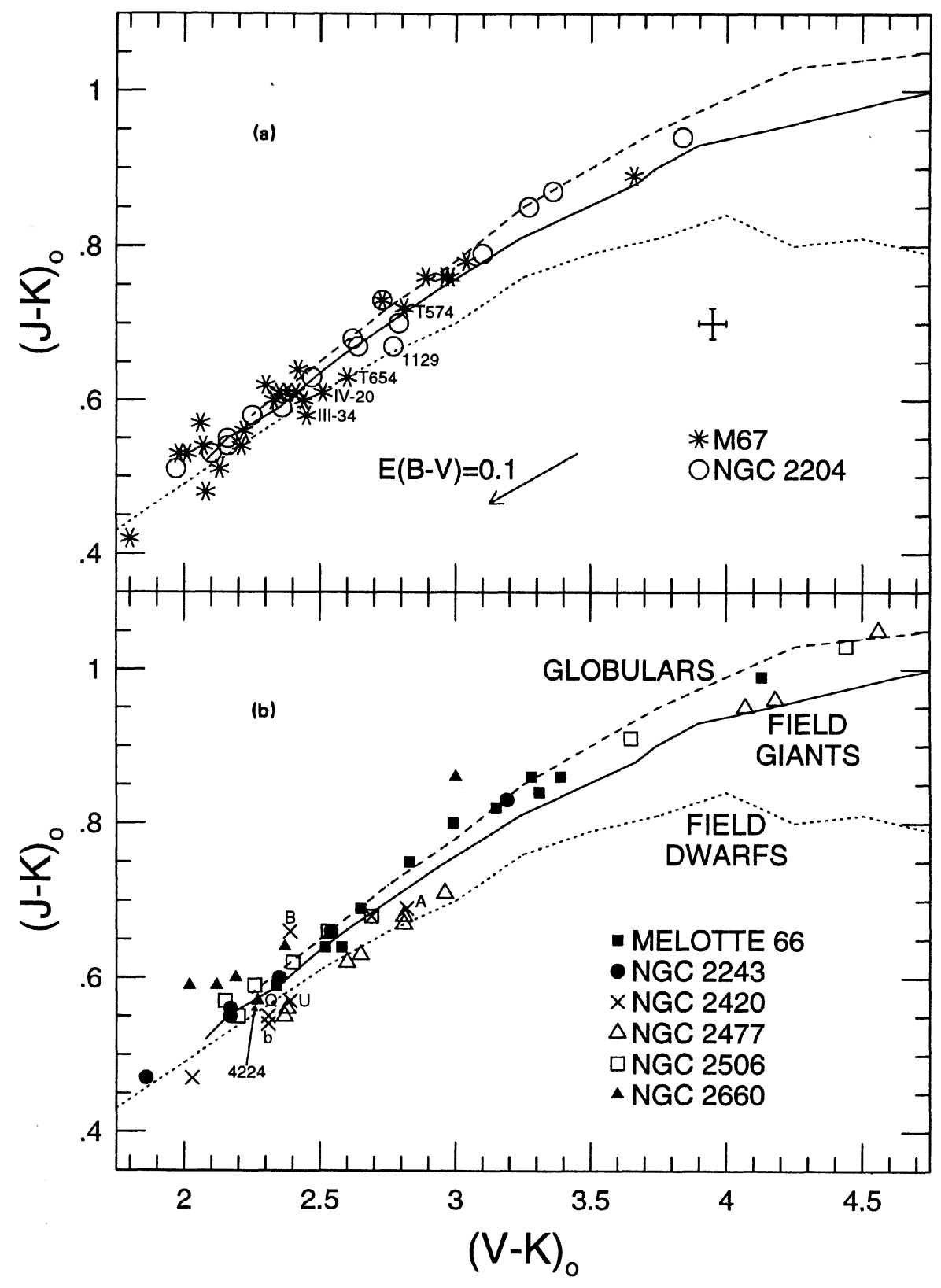

FIG. 10. $(J-K)_{0},(V-K)_{0}$ colors of the open cluster giants. Star 2215 in Melotte 66, stars F94 and F115 in M67, stars 1136 and 4132 in NGC 2204, stars C2 and 1220 in NGC 2477, and star 9009 in NGC 2660 are not shown. Interesting stars discussed in the text are individually labeled. The field relations are taken from Table 12 of Frogel et al. (1978); the globular cluster relation represents the mean values for M3, M13, and M92 and is taken from Fig. 2(c) of CFP. The error bars represent the $\pm 1 \sigma$ uncertainties in the observed colors; the effect of increasing the value of $\mathrm{E}(B-V)$ by $0.1 \mathrm{mag}$ is also shown. 
The $V$ magnitudes of stars in NGC 2420 presented by Sarma \& Walker (1962), West (1967), McClure et al. (1974), McClure et al. (1978), and Anthony-Twarog et al. (1990) are all in relatively good agreement. Thus, the systematic offset of stars $\mathrm{A}, \mathrm{Q}, \mathrm{U}$, and $\mathrm{b}$ from the field line in Fig. 10 (b) suggests that either $J$ is systematically too bright by $0.03 \mathrm{mag}$ or $K$ is systematically too faint by $0.04 \mathrm{mag}$. While all of the $J-K$ colors for this cluster have random uncertainties of $0.03 \mathrm{mag}$ or greater, there is no direct evidence for systematic errors in the infrared photometry, and we leave the cluster's locus as shown in Fig. 10(b).

Although there is no overall systematic deviation between our data and the combined infrared photometry of NGC 2477 and NGC 2660 presented by BWLE, the $J-K$ colors of each individual cluster show systematic differences of just the right magnitude to produce the observed offsets from the field line in Fig. 10(b). However, these deviations are well within the photometric uncertainties of the two datasets, so we have assumed that our infrared values are correct. Since no errors can be identified in the $V$ photometry of these two clusters, the optical data have been used as reported in the literature, and the observed offsets of NGC 2477 and NGC 2660 in Fig. 10(b) remain.

\subsection{The JHK Colors and CO Indices}

Figure 11 presents the $(J-H)_{0},(H-K)_{0}$ diagrams for the giants in the eight open clusters we observed. The interpretation of these plots is complicated by the effects of $\mathrm{H}_{2} \mathrm{O}$ absorption (especially for very cool stars) and the uncertainties in the definition of the field giant relation [especially near the blue end in $(\mathrm{H}-\mathrm{K})_{0}$ ]; see FPC83 for a discussion of each of these effects. Although there are few stars with red enough $J H K$ colors to lie in the region where field and globular cluster giants are clearly separated, those that do generally scatter about the field giant line rather than the line defined by the globular cluster giants. It is apparent in Fig. 11(a) that M67's giants show considerable scatter. This could be the result of a high incidence of binaries in the cluster or the possible peculiarities previously mentioned in Sec. 4.2.

Obviously anomalous stars in Fig. 11 are B in NGC 2420

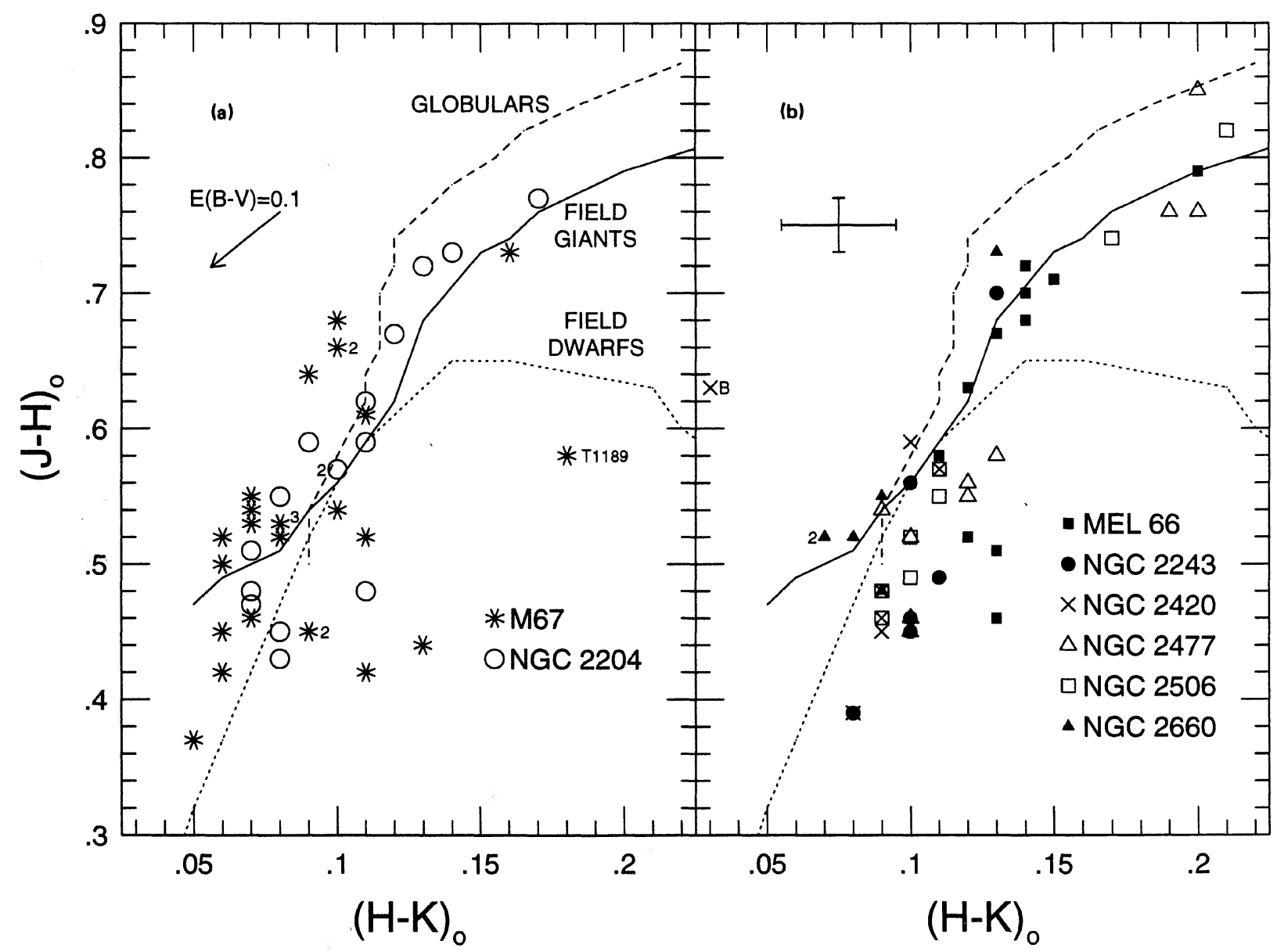

Fig. 11. $(J-H)_{0},(H-K)_{0}$ colors of the open cluster giants. Star 2215 in Melotte 66, stars F94 and F115 in M67, stars 1136 and 4132 in NGC 2204, stars C2 and 1220 in NGC 2477, and star 9009 in NGC 2660 are not shown. Interesting stars discussed in the text are individually labeled. Single digits near a symbol indicate the number of stars from a particular cluster represented by that symbol. The field relations are taken from Table 12 of Frogel et al. (1978); the globular cluster line represents the mean values for M3, M13, and M92 and is taken from Fig. 3(a) of CFP. The error bars represent the $\pm 1 \sigma$ uncertainties in the observed colors; the effect of increasing the value of $\mathrm{E}(B-V)$ by $0.1 \mathrm{mag}$ is also shown. 
and T1189 in M67. B has already been extensively discussed in Sec. 4.5, but T1189 does not appear unusual in any other color-color plot or in the cluster CMD (Fig. 2), nor does it have strong $\mathrm{H}_{2} \mathrm{O}$ absorption. For this reason, an error in $H-K$ is suspected for this star.

The CO measurements are displayed in Fig. 12. In general, each cluster follows a relatively tight distribution in $\mathrm{CO}$ as a function of $(V-K)_{0}$; discrepant stars were reviewed in Sec. 4. These CO indices will be discussed further in Sec. 6.

\section{THE CLUSTER FIDUCIAL PARAMETERS}

Using the procedure described in CFP and the Appendix of FPC81, a bolometric correction to the $K$ magnitude $B C_{K}$, a surface gravity $g$, and an effective temperature $T_{e}$ have been determined for each star with infrared observations.
These parameters are given in Table 10, and the resulting $H$ $R$ diagrams of the eight clusters appear in Figs. 13 and 14 . Our best estimates of the cluster giant branches are shown as dotted lines in these figures; the derivation of these GBs will be discussed below.

Also given in Table 10 is the mass used in determining the stellar parameters of the giant stars in each cluster. This mass was derived from the appropriate GB of the Revised Yale Isochrones using the following procedure:

(1) The GB mass of each particular isochrone at $\boldsymbol{M}_{\mathrm{bol}}$ $=-1.0, \mathscr{M}$ (iso), was determined by linear interpolation between the two isochrone points which bound $M_{\text {bol }}$ $=-1.0 . \mathscr{M}$ (iso) was derived for the isochrones which have a helium mass fraction $(Y)$ of 0.2 or 0.3 , a metals mass fraction $(Z)$ of $0.001,0.004,0.01$, or 0.04 , and an age $(\tau)$ of $0.5,0.75,1.0,3.0,5.0,7.0$, or $10.0 \mathrm{Gyr}$.

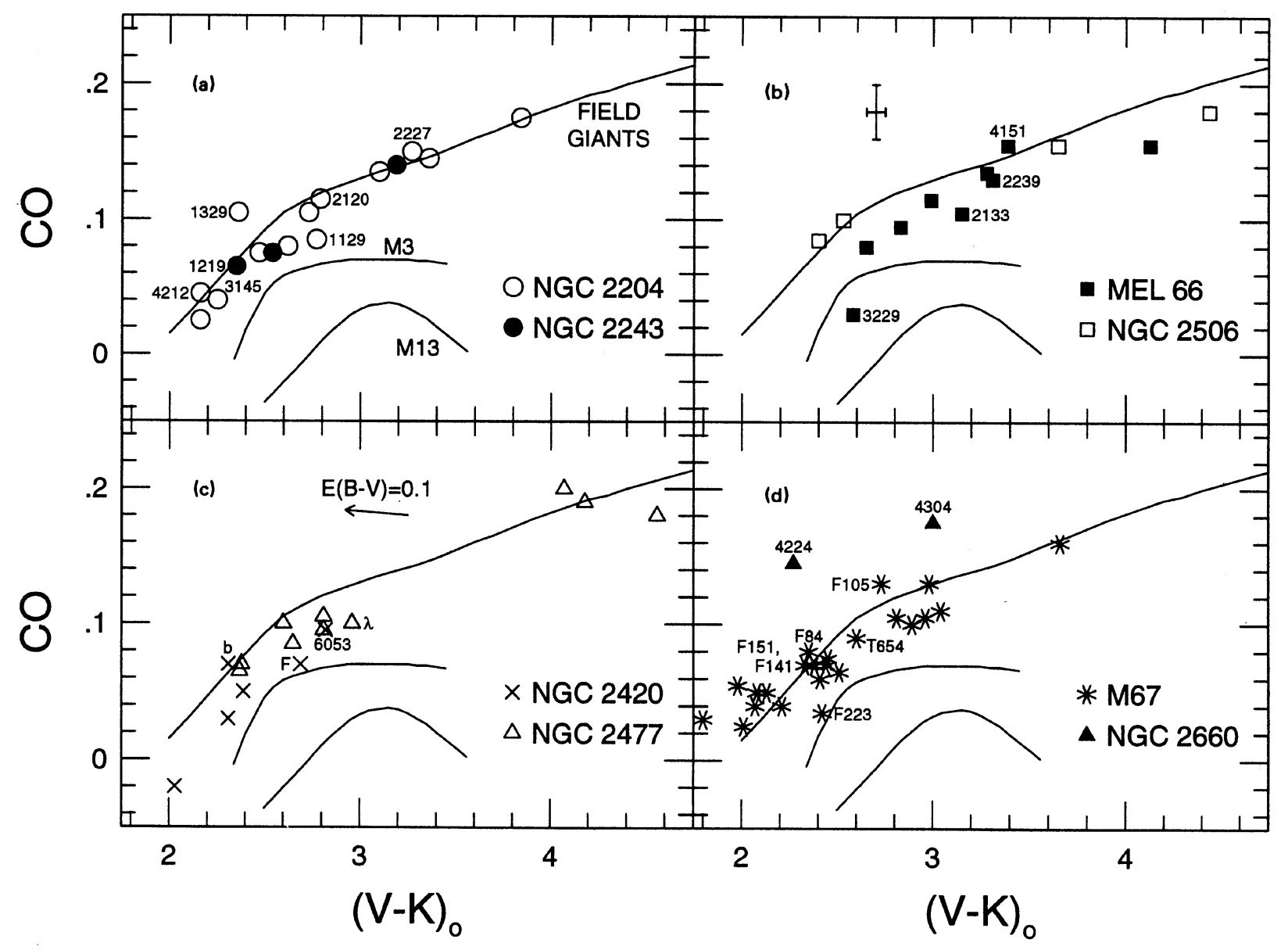

FIG. 12. CO indices of the open cluster giants. Star 2215 in Melotte 66, stars F94 and F115 in M67, stars 1136 and 4132 in NGC 2204 , stars C2 and 1220 in NGC 2477, and star 9009 in NGC 2660 are not shown. Interesting stars discussed in the text are individually labeled. The field giant relation is a smoothed version of that presented in Table 12 of Frogel et al. (1978); the M3 and M13 curves are taken from Fig. 1 (a) of CFP. The error bars represent the $\pm 1 \sigma$ uncertainties in the observed colors; the effect of increasing the value of $\mathrm{E}(B-V)$ by 0.1 mag is also shown. 
TABLE 10. Derived stellar parameters.

\begin{tabular}{|c|c|c|c|c|c|c|c|c|c|}
\hline Star & $\mathrm{BC}_{K}$ & $M_{\mathrm{bol}}$ & $\log T_{e}$ & $\log g$ & Star & $\mathrm{BC}_{K}$ & $M_{\mathrm{bol}}$ & $\log T_{e}$ & $\log g$ \\
\hline \multicolumn{5}{|c|}{ Melotte $66\left(1.1 \mathrm{M}_{\odot}\right)$} & \multicolumn{5}{|c|}{ M67 (Continued) } \\
\hline 2133 & 2.40 & -1.06 & 3.614 & 1.6 & T574 & 2.25 & -0.25 & 3.632 & 2.0 \\
\hline 4151 & 2.48 & -1.72 & 3.601 & 1.2 & $\mathrm{~T} 626^{\mathrm{b}}$ & 2.46 & -1.20 & 3.604 & 1.5 \\
\hline $1242^{\mathrm{a}}$ & 2.45 & -1.46 & 3.607 & 1.4 & T654 & 2.15 & +0.36 & 3.645 & 2.3 \\
\hline 2206 & 2.34 & -1.52 & 3.625 & 1.4 & $\mathrm{~T} 829^{\mathbf{b}}$ & 2.33 & -0.83 & 3.623 & 1.8 \\
\hline 2215 & 3.06 & -3.85 & 3.514 & 0.0 & $\mathrm{~T} 856^{\mathrm{a}}$ & 2.32 & -0.51 & 3.623 & 1.9 \\
\hline $2217^{a}$ & 2.18 & +0.12 & 3.643 & 2.1 & $\mathrm{~T} 1189^{\mathrm{a}}$ & 2.30 & -0.18 & 3.627 & 2.0 \\
\hline 2239 & 2.45 & -2.53 & 3.609 & 0.9 & IV-202 & 2.56 & -1.97 & 3.593 & 1.2 \\
\hline 2261 & 2.12 & -0.29 & 3.655 & 2.0 & \multirow{3}{*}{\multicolumn{5}{|c|}{ NGC $2204\left(1.4 \mathrm{M}_{\odot}\right)$}} \\
\hline 2277 & 2.70 & -1.13 & 3.577 & 1.4 & & & & & \\
\hline 3213 & 2.02 & +0.60 & 3.665 & 2.4 & & & & & \\
\hline 3229 & 2.14 & +0.03 & 3.649 & 2.1 & $\begin{array}{l}1129 \\
1136\end{array}$ & $\begin{array}{l}2.22 \\
2.95\end{array}$ & $\begin{array}{l}-1.23 \\
-3.24\end{array}$ & $\begin{array}{l}3.638 \\
3.539\end{array}$ & $\begin{array}{l}1.7 \\
0.5\end{array}$ \\
\hline $4229^{a}$ & 2.27 & -0.41 & 3.632 & 1.9 & 2120 & 2.23 & -2.29 & 3.640 & 1.3 \\
\hline \multirow{2}{*}{\multicolumn{5}{|c|}{$\operatorname{M67}\left(1.3 \mathrm{M}_{\odot}\right)$}} & 2136 & 2.09 & -0.71 & 3.658 & 2.0 \\
\hline & & & & & 3145 & 1.97 & -0.56 & 3.676 & 2.1 \\
\hline F84 & 2.03 & +0.52 & 3.663 & 2.5 & 4132 & 2.88 & -4.36 & 3.552 & 0.1 \\
\hline F94 & 1.34 & +3.17 & 3.731 & 3.8 & $4137^{a}$ & 2.46 & -2.53 & 3.605 & 1.0 \\
\hline $\mathrm{F} 105^{\mathrm{a}}$ & 2.22 & +0.08 & 3.636 & 2.2 & 2212 & 2.16 & -1.09 & 3.649 & 1.8 \\
\hline $\mathrm{F} 108^{\mathrm{a}}$ & 2.35 & -0.69 & 3.619 & 1.8 & 2222 & 2.17 & -0.04 & 3.645 & 2.2 \\
\hline F115 & 1.41 & +2.97 & 3.724 & 3.7 & 2227 & 2.43 & -0.98 & 3.606 & 1.7 \\
\hline F117 & 1.80 & +2.77 & 3.677 & 3.4 & 3215 & 1.92 & +0.09 & 3.679 & 2.4 \\
\hline F141 & 2.05 & +0.43 & 3.661 & 2.4 & 4210 & 1.89 & +0.18 & 3.682 & 2.4 \\
\hline F151 & 2.02 & +0.47 & 3.665 & 2.4 & 4212 & 1.92 & -1.27 & 3.685 & 1.9 \\
\hline F164 & 2.06 & +0.46 & 3.659 & 2.4 & 4223 & 1.83 & +0.33 & 3.691 & 2.5 \\
\hline $\mathrm{F} 170^{\mathrm{a}}$ & 2.32 & -0.69 & 3.622 & 1.8 & 1329 & 2.04 & -2.22 & 3.672 & 1.4 \\
\hline F193 & 1.96 & +2.31 & 3.667 & 3.2 & $3304^{a}$ & 2.36 & -1.85 & 3.619 & 1.4 \\
\hline F223 & 2.08 & +0.51 & 3.658 & 2.4 & $3324^{\mathrm{a}}$ & 2.23 & -1.06 & 3.640 & 1.8 \\
\hline F224 & 2.07 & +0.69 & 3.655 & 2.5 & $3325^{\mathrm{a}}$ & 2.62 & -3.17 & 3.586 & 0.7 \\
\hline F227 & 1.88 & +3.09 & 3.672 & 3.5 & \multirow{2}{*}{\multicolumn{5}{|c|}{ NGC $2243\left(1.2 \mathrm{M}_{\odot}\right)$}} \\
\hline F231 & 2.02 & +1.52 & 3.664 & 2.9 & & & & & \\
\hline F244 & 1.88 & +0.87 & 3.681 & 2.7 & $4110^{a}$ & 2.12 & -0.59 & 3.653 & 1.9 \\
\hline I-17 & 1.95 & +2.43 & 3.666 & 3.2 & 1219 & 2.04 & -1.55 & 3.672 & 1.6 \\
\hline II-22 & 1.86 & +3.01 & 3.670 & 3.5 & $4209^{a}$ & 2.41 & -1.80 & 3.614 & 1.3 \\
\hline III-34 & 2.06 & +1.22 & 3.652 & 2.7 & 2308 & 1.93 & +0.34 & 3.679 & 2.4 \\
\hline IV-20 & 2.10 & +1.09 & 3.649 & 2.6 & 4301 & 1.75 & +1.03 & 3.697 & 2.8 \\
\hline IV-68 & 1.84 & +3.07 & 3.674 & 3.5 & 4303 & 1.92 & +0.43 & 3.678 & 2.4 \\
\hline IV-77 & 1.90 & +2.95 & 3.668 & 3.4 & & & & & \\
\hline IV-81 & 1.70 & +2.98 & 3.690 & 3.5 & & & & & \\
\hline
\end{tabular}


TABLE 10. (continued)

\begin{tabular}{|c|c|c|c|c|c|c|c|c|c|}
\hline Star & $\mathrm{BC}_{K}$ & $M_{\mathrm{bol}}$ & $\log \mathrm{T}_{\mathrm{e}}$ & $\log g$ & Star & $\mathrm{BC}_{K}$ & $M_{\text {bol }}$ & $\log \mathrm{T}_{\mathrm{e}}$ & $\log g$ \\
\hline \multicolumn{5}{|c|}{ NGC $2420\left(1.2 \mathrm{M}_{\odot}\right)$} & \multicolumn{5}{|c|}{ NGC $2506\left(1.3 \mathrm{M}_{\odot}\right)$} \\
\hline $\mathrm{A}^{\mathrm{a}}$ & 2.24 & -0.93 & 3.634 & 1.7 & 1112 & 1.92 & +0.34 & 3.679 & 2.4 \\
\hline B & 2.02 & +2.15 & 3.655 & 3.0 & 2122 & 2.12 & -1.06 & 3.655 & 1.8 \\
\hline $\mathrm{D}$ & 1.84 & -0.30 & 3.690 & 2.2 & 2212 & 2.05 & -0.78 & 3.664 & 1.9 \\
\hline $\mathrm{F}$ & 2.18 & -0.82 & 3.644 & 1.8 & $3254^{\mathrm{b}}$ & 2.43 & -2.08 & 3.610 & 1.2 \\
\hline$Q$ & 1.99 & +0.36 & 3.668 & 2.4 & 4205 & 1.94 & +0.65 & 3.675 & 2.6 \\
\hline $\mathrm{U}^{\mathrm{a}}$ & 2.03 & +0.17 & 3.662 & 2.3 & $4228^{b}$ & 2.68 & -1.77 & 3.578 & 1.2 \\
\hline$x^{b}$ & 2.19 & -0.96 & 3.645 & 1.8 & 4240 & 1.98 & +0.47 & 3.672 & 2.5 \\
\hline $\mathrm{b}$ & 1.99 & +0.44 & 3.667 & 2.4 & $2401^{a}$ & 2.56 & -2.38 & 3.593 & 1.0 \\
\hline \multirow[t]{3}{*}{ II- $3-44^{b}$} & 1.94 & +1.33 & 3.671 & 2.8 & $2402^{a}$ & 2.19 & -0.43 & 3.643 & 2.0 \\
\hline & \multirow{2}{*}{\multicolumn{4}{|c|}{ NGC $2477\left(1.9 \mathrm{M}_{\odot}\right)$}} & 4402 & 2.77 & -1.47 & 3.566 & 1.3 \\
\hline & & & & & \multicolumn{5}{|c|}{ NGC $2660\left(2.1 \mathrm{M}_{\odot}\right)$} \\
\hline 1044 & 2.04 & +0.23 & 3.660 & 2.5 & & & & & \\
\hline $1069^{a}$ & 2.68 & -2.86 & 3.578 & 0.9 & 9009 & 3.22 & -5.34 & 3.520 & -0.3 \\
\hline $2009^{a}$ & 2.24 & -0.87 & 3.633 & 1.9 & $9016^{a}$ & 1.96 & +0.08 & 3.676 & 2.5 \\
\hline 4035 & 2.80 & -1.17 & 3.562 & 1.5 & 9023 & 1.93 & -0.38 & 3.682 & 2.4 \\
\hline \multirow[t]{2}{*}{$4067^{a}$} & 2.16 & -0.36 & 3.643 & 2.2 & 9025 & 2.06 & -1.00 & 3.667 & 2.1 \\
\hline & & & & & 2121 & 1.87 & -0.83 & 3.690 & 2.2 \\
\hline $6053^{a}$ & 2.24 & -0.78 & 3.633 & 2.0 & & & & & \\
\hline 8019 & 2.03 & +0.62 & 3.659 & 2.6 & 4224 & 2.02 & -1.93 & 3.676 & 1.7 \\
\hline $2117^{a}$ & 2.70 & -2.72 & 3.575 & 1.0 & $4304^{a}$ & 2.37 & -2.47 & 3.625 & 1.3 \\
\hline$\theta^{\mathrm{a}}$ & 2.14 & -0.48 & 3.646 & 2.2 & & & & & \\
\hline$\lambda$ & 2.30 & -0.64 & 3.623 & 2.0 & & & & & \\
\hline 1220 & 3.21 & -1.68 & 3.518 & 1.2 & & & & & \\
\hline $\mathrm{C} 2$ & 3.17 & -4.08 & 3.522 & 0.2 & & & & & \\
\hline
\end{tabular}

a These stars were given the greatest weight in defining the giant branch and were the only stars used in determining $\mathrm{CO}(\mathrm{GB})$ for the respective cluster.

${ }^{b}$ Proper motion nonmember; parameters may not be representative if the star and cluster do not share the same reddening and/or distance modulus. 
(2) Linear regressions ${ }^{5}$ were performed to derive relations between $\log \mathscr{M}$ (iso) and $\log \tau$ at each isochrone metallicity and helium abundance.

(3) For a given cluster, $\mathscr{M}$ (iso) was calculated from the linear relations of step (2) for the isochrones bounding the cluster metallicity at each $Y$. To determine the relevant isochrone metallicities, the cluster's $[\mathrm{Fe} / \mathrm{H}]$ value in Table 1 was converted to $Z$ assuming $Z_{\odot}=0.02$.

(4) Linear interpolation in $Z$ then gave a mass appropriate to the cluster at each helium abundance.

(5) The arithmetic mean of the $Y=0.2$ and $Y=0.3$ results was the GB mass assumed as characteristic of the cluster giants.

Combining the stellar data of Table 10 and the infrared photometry, various fiducial parameters were derived which characterize the GB of each open cluster. The first step in this procedure was the delineation of the cluster GB in the $H-R$ diagram. Stars with uncertain photometry and those suspected of being variables, binaries, AGB stars, or nonmembers (see discussion in Sec. 4) were given lower weight or excluded entirely. The stars given the greatest probability of being cluster GB stars are noted in Table 10. Other factors were also considered, including the location of the clump stars and the slopes of the more precisely determined 47 Tuc, M3, and M92 GBs shown in Figs. 13 and 14. Because the presence of AGB stars and undetected binaries could not be ruled out, the final GB (drawn by hand) was biased slightly to cooler temperatures when its position was somewhat ambiguous.

Once the GB had been defined, each cluster's fiducial parameters were determined; the results are presented in Table 11. $M_{\text {bol }}(\mathrm{GB})$ is the bolometric magnitude of the cluster's GB at $(J-K)_{0}=0.7 ;(J-K)_{\mathrm{GB}}$ is the GB color and $\log T_{e}(\mathrm{~GB})$ is the GB temperature, each taken at $M_{\mathrm{bol}}$ $=-1$.0. $\mathrm{CO}(\mathrm{GB}), \sigma(\mathrm{CO})$, and $\log T_{e}$ (iso) will be discussed below. Based upon those clusters whose $G B$ positions are the most uncertain, it is conservatively estimated that the random error due only to positioning the GB produces uncertainties in the derived parameters of $\pm 0.30 \mathrm{mag}$ in $M_{\text {bol }}(\mathrm{GB}), \pm 0.03 \mathrm{mag}$ in $(J-K)_{\mathrm{GB}}$, and \pm 0.005 in $\log T_{e}$ (GB).

Columns 3 and 4 of Table 12 illustrate the mean uncertainties in a star's effective temperature and bolometric correction due to the estimated uncertainties in the data used to calculate each. These uncertainties were derived by changing the value of the specific parameter given in column 1 by its representative uncertainty (column 2) for every star in NGC 2204 and averaging the resultant changes in $\log T_{e}$ and $M_{\text {bol }}$. Because $\log T_{e}$ (GB) will change if the GB is shifted in either temperature or luminosity, each uncertainty in $M_{\text {bol }}$ was converted to a respective uncertainty in $\log T_{e}$ (GB) using the slope of the 47 Tuc GB in the $H-R$ diagram. The total uncertainty in $\log T_{e}(\mathrm{~GB})$ due to an uncertainty in a single parameter is given in the final column of Table 12 and is simply the sum of the direct uncertainty in effective temperature and that due to an uncertainty in $\boldsymbol{M}_{\text {bol }}$. Summing all of the uncertainties in Table 12 in quadrature, we find an overall uncertainty of \pm 0.023 in $\log T_{e}(\mathrm{~GB})$.

${ }^{5}$ For all linear relations discussed in this paper, the regression has been performed using each parameter as the independent variable. The average of the resulting relations has been used in the calculations and/or quoted in the text. Also, the uncertainties in the coefficients are given at the $\pm 1 \sigma$ level, and cluster age is always expressed in Gyr.

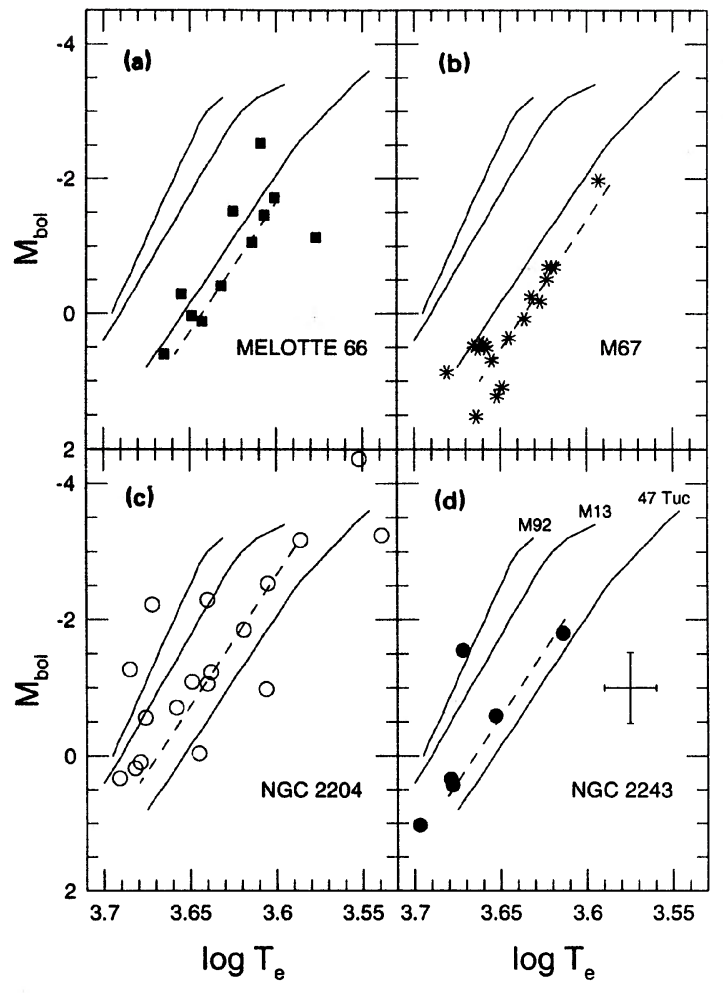

FIG. 13. $H-R$ diagrams of Melotte 66, M67, NGC 2204, and NGC 2243. Star 2215 (near the GB tip) in Melotte 66 and stars F94, F115, F117, F193, F227, I-17, II-22, IV-68, IV-77, and IV-81 (on the lower GB and subgiant branch) in M67 are not shown. The giant branches of M3, M92, and 47 Tucanae (taken from Table 8 of FPC81) are presented for comparison. The error bars represent the $\pm 1 \sigma$ uncertainties in $\log T_{e}$ and $M_{\text {bol }}$ determined from Table 12.

With the data in Table 12 and the 47 Tucanae photometry and bolometric magnitudes from FPC81, the uncertainties in $M_{\mathrm{bol}}(\mathrm{GB})$ and $(J-K)_{\mathrm{GB}}$ can be similarly examined. Doing so, the total estimated uncertainty in $M_{\mathrm{bol}}(\mathrm{GB})$ is $\pm 0.62 \mathrm{mag}$, and that in $(J-K)_{\mathrm{GB}}$ is $\pm 0.07 \mathrm{mag}$.

For the globular clusters in FCP, the fiducial parameter $\mathrm{CO}(\mathrm{GB})$ is defined to be the median $\mathrm{CO}$ value of the stars with $(V-K)_{0}$ in the neighborhood of $3.0 \mathrm{mag}$. In order to compare the $\mathrm{CO}$ measurements of open clusters with those of globular clusters, we should define $\mathrm{CO}(\mathrm{GB})$ similarly here. Unfortunately, the number of open cluster giants with a $(V-K)_{0}$ color near $3.0 \mathrm{mag}$ is small (see Fig. 12), and those that do are often peculiar or displaced from the cluster GB in the CMD. Thus, we have determined $\mathrm{CO}(\mathrm{GB})$ in a slightly different manner for the open clusters than was done for the globulars.

Persson et al. (1980) define the parameter $R(\mathrm{CO})$, a star's $\mathrm{CO}$ absorption normalized with respect to the $\mathrm{CO}$ index predicted for a blackbody of similar $(V-K)_{0}$ but of solar composition. We have assumed that $R(\mathrm{CO})$ is the same for any star of a given metallicity, regardless of its color (i.e., its $T_{e}$ ). For each star noted in Table 10 as a likely GB star, we then determined the $\mathrm{CO}$ index appropriate to its $R(\mathrm{CO})$ value if its $(V-K)_{0}=3.0 . \mathrm{CO}(\mathrm{GB})$ is the median of these 


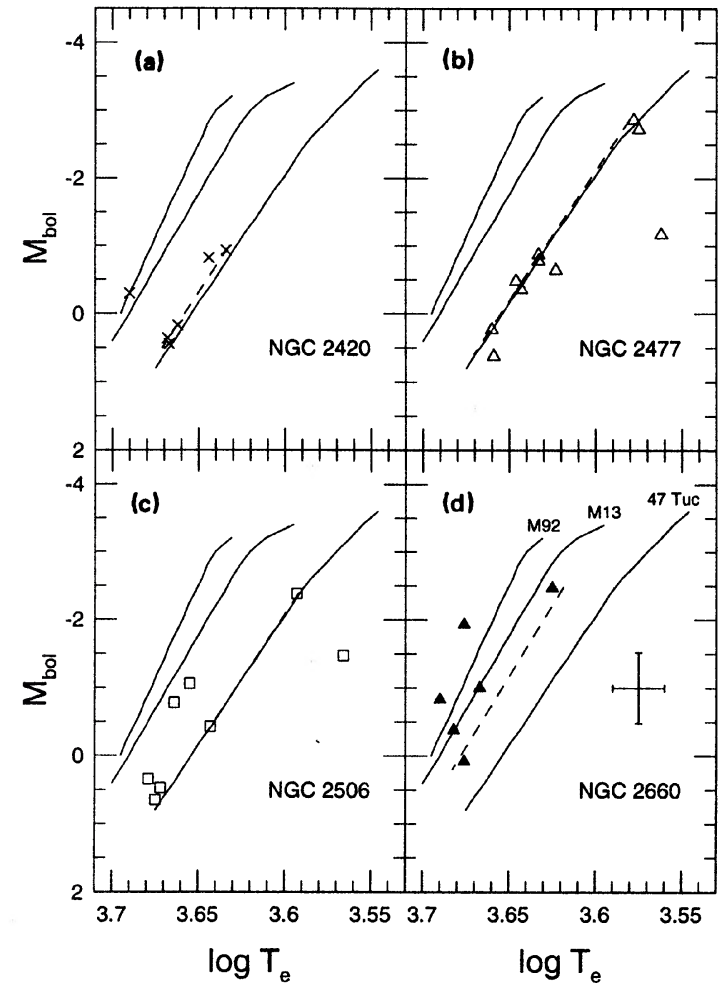

FIG. 14. $H-R$ diagrams of NGC 2420, NGC 2477, NGC 2506, and NGC 2660. Star B (a probable nonmember) in NGC 2420 and the three carbon stars (C2 and 1220 in NGC 2477, and 9009 in NGC 2660) are not shown. See the caption of Fig. 13 for further details.

$\mathrm{CO}$ indices for a given cluster and is listed in column 5 of Table 11 .

$\sigma(\mathrm{CO})$, column 6 of Table 11 , is the estimated uncertainty in $\mathrm{CO}(\mathrm{GB})$. It was derived by converting the $R(\mathrm{CO})$ ratio of each of the stars observed in a cluster to the corresponding $\mathrm{CO}$ index at $(V-K)_{0}=3.0$ and taking the standard deviation of these values about $\mathrm{CO}(\mathrm{GB})$; only stars with $(V$ $K)_{0}<2.0$, the carbon stars, and the barium stars were excluded in determining $\sigma(\mathrm{CO})$. Since known nonmembers have been included in this calculation, it yields a conservative upper limit to the true value of $\sigma(\mathrm{CO})$.

Figure 15 shows a plot of $[\mathrm{Fe} / \mathrm{H}]$ vs $\mathrm{CO}(\mathrm{GB})$ for the open clusters (taking $[\mathrm{Fe} / \mathrm{H}]$ from Table 1 ) and for globular clusters (FCP). For the open clusters, there is no significant correlation (neither linear nor rank ordered) between $[\mathrm{Fe} / \mathrm{H}]$ and $\mathrm{CO}(\mathrm{GB})$. Undoubtedly, this is due to the limited range in $[\mathrm{Fe} / \mathrm{H}]$ and $\mathrm{CO}(\mathrm{GB})$ spanned by the open clusters, the small size of the sample in terms of both number of clusters and number of stars in each cluster, and the fact that there may be significant errors in the optical determinations of $[\mathrm{Fe} / \mathrm{H}]$. Nevertheless, the open clusters do overlap the globular clusters of FCP that have a similar range in $[\mathrm{Fe} / \mathrm{H}]$ and $\mathrm{CO}(\mathrm{GB})$. A linear regression of the combined data has a correlation coefficient $r=0.91$ and yields

$$
\begin{aligned}
{[\mathrm{Fe} / \mathrm{H}]_{\mathrm{CO}}=} & -2.26( \pm 0.25) \\
& +14.99( \pm 0.87) \mathrm{CO}(\mathrm{GB}) .
\end{aligned}
$$

This new metallicity index is easily determined for any cluster by simple photometric measurements of a sample of its GB stars. We propose that it is valid for all star clusters over a range of $2.5 \mathrm{dex}$ in $[\mathrm{Fe} / \mathrm{H}]$ and for ages from $10^{8}$ to $10^{10} \mathrm{yr}$. The addition of the open clusters improves the determination of $[\mathrm{Fe} / \mathrm{H}]$ from $\mathrm{CO}$ values for stars of solar metallicity and increases our confidence in its use for stars in the galactic bulge.

It should be noted that Eq. (1) predicts that $\mathrm{CO}(\mathrm{GB})=0.151$ for a cluster of solar metallicity. However, a giant with this CO strength and $(V-K)_{0}=3.00$ will lie above the field giant relation of Fig. 12. This may be an indication that the stars used to determine the field giant lines in the color-color diagrams (Figs. 9-12) have an average metallicity which is less than solar. Although this could imply that the absolute reddening values we have chosen for M67 and NGC 2477 are incorrect (see Sec. 5.1), the reduced scatter in the infrared CMD of NGC 2477 (Fig. 7) suggests that our procedure has properly accounted for the relative reddening differences among this cluster's stars.

\section{AN AGE-METALLICITY RELATION FOR OPEN CLUSTERS}

Although our cluster sample is small, we note linear correlations at greater than $90 \%$ confidence between the average literature metallicity in Table 1 and three other cluster parameters - age, distance above or below the galactic plane, and galactocentric distance. The age-metallicity relation exhibited by the eight open clusters is

$$
[\mathrm{Fe} / \mathrm{H}]=0.11( \pm 0.19)-0.135( \pm 0.052) \tau
$$

and is significant at the $90 \%$ confidence level $(r=0.63)$.

Figure 16(a) compares the age-metallicity relation of the galactic open clusters with those of Large Magellanic Cloud (LMC) clusters and stars in the solar neighborhood. The LMC cluster data are taken from Cohen (1982) and Mateo (1988), the solar neighborhood relation is that of Carlberg et al. (1985), and the dashed line represents Eq. (2). If our cluster sample is representative of the entire galactic open cluster system, then Fig. 16(a) shows that the heavy metal enrichment of this system was similar to that of the LMC but lagged that of the solar neighborhood, as also proposed by Geisler (1987).

However, theories of galactic formation generally predict that the relationship between metallicity and galactocentric radius is more fundamental than other metallicity correlations which may exist. Thus, we have adjusted the average literature $[\mathrm{Fe} / \mathrm{H}]$ values of the open clusters to those appropriate to the solar radius $(8.5 \mathrm{kpc})$, using the galactocentric distances given in Table 1 and the galactic radial metallicity gradient $(-0.14 \mathrm{dex} / \mathrm{kpc})$ derived by Janes et al. (1988) from a much larger sample of old open clusters. After doing this, age and metallicity remain correlated (now at the $92 \%$ confidence level), and linear regression gives

$$
[\mathrm{Fe} / \mathrm{H}]=0.17( \pm 0.13)-0.090( \pm 0.032) \tau \text {. }
$$

This new relation is shown as the dashed line in Fig. 16(b), and it now appears that the metal enrichment of the old open clusters was intermediate to those of the LMC and the solar neighborhood, assuming that an analogous radial metallicity gradient of some type does not exist in the LMC. This may be an indication that the old open cluster giants are akin to stars of the galactic thick disk; a similar conclusion has been reached by Lynga (1987). Figure 16 and the above discussion illustrate the caution that must be applied in the 
TABLE 11. Cluster giant branch parameters.

\begin{tabular}{|c|c|c|c|c|c|c|}
\hline \multirow{2}{*}{ Cluster } & \multicolumn{5}{|c|}{ Empirical Parameters } & \multirow{2}{*}{$\begin{array}{c}\log \mathrm{T}_{e} \\
\text { (iso) }\end{array}$} \\
\hline & $M_{\mathrm{bol}}(\mathrm{GB})$ & $(J-K)_{\text {GB }}$ & $\log \mathrm{T}_{\mathrm{e}}(\mathrm{GB})$ & $\mathrm{CO}(\mathrm{GB})$ & $\sigma(\mathrm{CO})$ & \\
\hline Melotte 66 & +0.03 & 0.81 & 3.617 & 0.102 & 0.026 & 3.625 \\
\hline M67 & +0.19 & 0.82 & 3.610 & 0.118 & 0.031 & 3.611 \\
\hline NGC 2204 & -0.94 & 0.71 & 3.643 & 0.129 & 0.024 & 3.628 \\
\hline NGC 2243 & -0.84 & 0.72 & 3.639 & 0.117 & 0.016 & 3.634 \\
\hline NGC 2420 & -1.03 & 0.70 & 3.632 & 0.096 & 0.028 & 3.627 \\
\hline NGC 2477 & -0.95 & 0.71 & 3.629 & 0.119 & 0.013 & 3.623 \\
\hline NGC 2506 & -0.60 & 0.75 & 3.628 & 0.125 & 0.011 & 3.632 \\
\hline NGC $2660^{a}$ & -0.92 & 0.71 & 3.654 & $\ldots$ & $\ldots$ & 3.633 \\
\hline
\end{tabular}

${ }^{a} \mathrm{CO}(\mathrm{GB})$ was not determined for NGC 2660 . Star 4304 was the only GB star observed in the CO band, and it may be peculiar (see Sec. 4.8).

TABLE 12. Error propagation.

\begin{tabular}{|c|c|c|c|c|}
\hline \multirow{2}{*}{ Parameter } & \multirow{2}{*}{ Uncertainty } & \multicolumn{3}{|c|}{ Uncertainties Produced ${ }^{a}$} \\
\hline & & $\mathrm{BC}_{K}$ & $\log \mathrm{T}_{\mathrm{e}}$ & $\log \mathrm{T}_{\mathrm{e}}(\mathrm{GB})$ \\
\hline $\mathrm{E}(B-V)$ & $\pm 0.05 \mathrm{mag}$ & $\mp 0.09$ & \pm 0.010 & \pm 0.012 \\
\hline$(m-M)_{\mathrm{O}}$ & $\pm 0.5 \mathrm{mag}$ & $\mp 0.50$ & \pm 0.002 & \pm 0.015 \\
\hline GB Mass & $\pm 0.5 \mathrm{M}_{\odot}$ & none & $\mp 0.001$ & $\mp 0.001$ \\
\hline$U$ & $\pm 0.05 \mathrm{mag}$ & none & none & none \\
\hline$B$ & $\pm 0.05 \mathrm{mag}$ & none & none & none \\
\hline$V$ & $\pm 0.05 \mathrm{mag}$ & \pm 0.01 & $\mp 0.003$ & $\mp 0.003$ \\
\hline$R_{\mathrm{C}}$ & $\pm 0.05 \mathrm{mag}$ & \pm 0.01 & none & none \\
\hline$J$ & $\pm 0.02 \mathrm{mag}$ & \pm 0.01 & none & none \\
\hline$H$ & $\pm 0.02 \mathrm{mag}$ & none & none & none \\
\hline$K$ & $\pm 0.02 \mathrm{mag}$ & none & \pm 0.001 & \pm 0.001 \\
\hline$L$ & $\pm 0.02 \mathrm{mag}$ & none & none & none \\
\hline $\mathrm{CO}$ & $\pm 0.02 \mathrm{mag}$ & none & none & none \\
\hline $\mathrm{H}_{2} \mathrm{O}$ & $\pm 0.02 \mathrm{mag}$ & none & none & none \\
\hline $\mathrm{T}_{\mathrm{e}}^{\mathrm{b}}$ & $\pm 100 \mathrm{~K}$ & & \pm 0.010 & \pm 0.010 \\
\hline $\mathrm{BC}_{K}^{\mathrm{C}}$ & $\pm 0.10 \mathrm{mag}$ & \pm 0.10 & & $\mp 0.003$ \\
\hline GB Drawing & & & & \pm 0.005 \\
\hline
\end{tabular}

${ }^{\text {a }}$ Uncertainties are derived by adjusting a given parameter for every star in NGC 2204 and averaging the resultant changes in $\mathrm{BC}_{K}$ and $\log T_{e}$. The change in $\log T_{e}$ (GB) due to a change in $M_{\mathrm{bol}}$ is estimated from the slope of the 47 Tuc giant branch in the $H-R$

${ }^{\text {diagram. }}$ The uncertainty in determining $T_{e}$ from $(V-K)_{0}$ is taken from CFP; the uncertainty in $\log T_{e}$ is given at $4500 \mathrm{~K}$.

c The uncertainty in determining $\mathrm{BC}_{K}$ from the photometry is taken from the appendix of FPC 81 . 


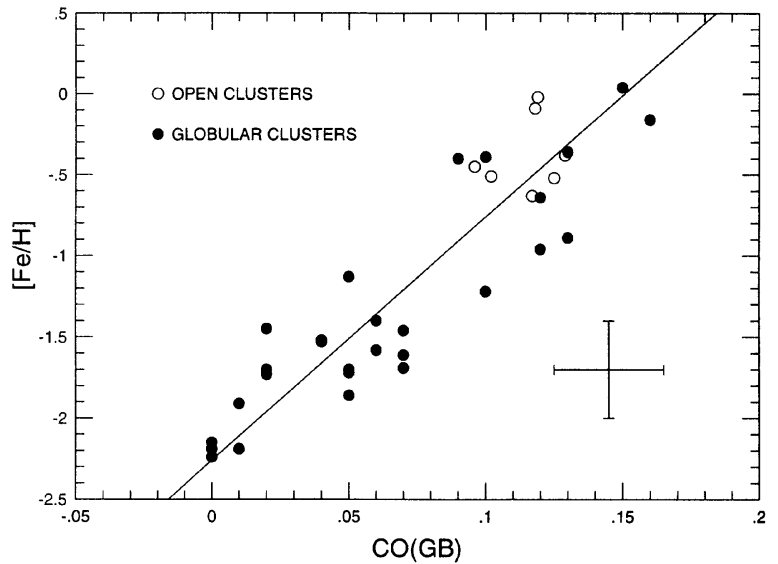

FIG. 15. Relationship between $[\mathrm{Fe} / \mathrm{H}]$ and $\mathrm{CO}(\mathrm{GB})$. The globular cluster data are taken from Table 1 of FCP; the open cluster values are taken from Tables 1 and 11. The solid line represents the best linear fit to the combined data. The error bars represent uncertainties of $\pm 0.3 \mathrm{dex}$ in $[\mathrm{Fe} / \mathrm{H}]$ (see Sec. 2) and \pm 0.021 in $\mathrm{CO}(\mathrm{GB})$, the average of $\sigma(\mathrm{CO})$ from Table 11.

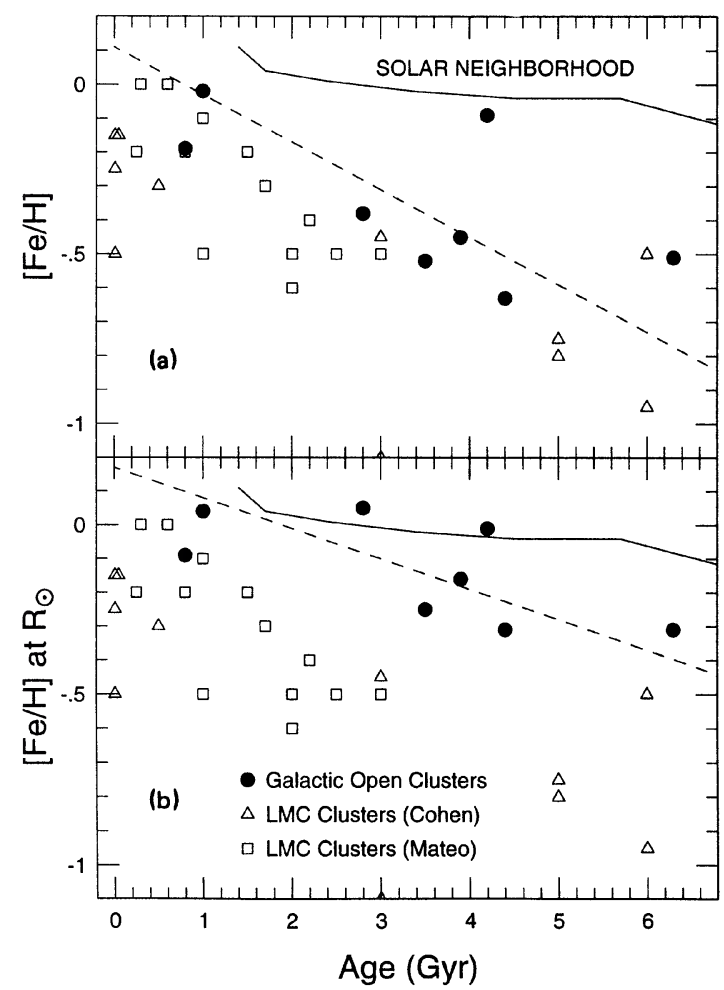

FIG. 16. Relationship between cluster metallicity and age. The LMC cluster data are taken from Tables 8 and 9 of Cohen (1982) and Fig. 2 of Mateo (1988); the solar neighborhood relation is taken from Carlberg et al. (1985). (a) The literature averages of $[\mathrm{Fe} / \mathrm{H}]$ given in Table 1 have been used for the open clusters. The dashed line represents the best linear fit to the open cluster data. (b) The literature averages of $[\mathrm{Fe} / \mathrm{H}]$ given in Table 1 have been normalized to a solar galactocentric distance of $8.5 \mathrm{kpc}$ using the open cluster galactocentric distances given in Table 1 and the radial galactic metallicity gradient of $-0.14 \mathrm{dex} / \mathrm{kpc}$ derived by Janes et al. (1988) from a large sample of open clusters with ages greater than $0.2 \mathrm{Gyr}$. The dashed line is again the best linear fit to the open cluster data. interpretation of correlations when more than one independent variable is involved.

\section{A COMPARISON OF THE CLUSTER GIANT BRANCHES WITH THE REVISED YALE ISOCHRONES}

By interpolating between the same isochrone points used to find $\mathscr{M}$ (iso) in Sec. 6, we obtained values for $\log T_{e}$ (iso), the effective temperature of the Revised Yale Isochrone GB at $M_{\text {bol }}=-1.0$. As expected, there is a tight linear correlation between $\log T_{e}$ (iso) and $\log \tau$ at a given $Z$ and between $\log T_{e}$ (iso) and $[\mathrm{Fe} / \mathrm{H}]$ at a given age. Surprisingly, though, the $Y=0.3$ isochrone temperatures are not systematically hotter than the $Y=0.2$ data; instead, helium abundance has only a small random effect on the isochrone GB temperature at $M_{\mathrm{bol}}=-1.0$. For this reason, $\log T_{e}$ (iso) can be characterized by age and metallicity alone. Linear regression of the isochrone data gives the theoretical relation between age, metallicity, and GB temperature:

$\log T_{e}$ (iso) $=3.622( \pm 0.027)-0.04470( \pm 0.00047)$

$$
\times[\mathrm{Fe} / \mathrm{H}]-0.02474( \pm 0.00030) \log \tau
$$

with a correlation coefficient $r>0.99$. For the remainder of this paper, we will designate as $\log T_{e}$ (iso) the temperature which results when a cluster's age and $[\mathrm{Fe} / \mathrm{H}]$ from Table 1 are substituted into Eq. (4). The final column of Table 11 presents $\log T_{e}$ (iso) for each of the open clusters we observed.

The empirically determined GB temperature given in Table $11, \log T_{e}(\mathrm{~GB})$, is also linearly correlated (at greater than $98 \%$ confidence) with $[\mathrm{Fe} / \mathrm{H}]$ and $\log \tau$ (both from Table 1), but the result differs significantly from the theoretical relation given above. This empirical relation is

$\log T_{e}(\mathrm{~GB})=3.634( \pm 0.439)-0.06900( \pm 0.01174)$

$$
\times[\mathrm{Fe} / \mathrm{H}]-0.06022( \pm 0.00960) \log \tau(5)
$$

with an rms deviation of 0.006 between the individually determined, empirical $\log T_{e}$ (GB) values and those predicted by Eq. (5).

An examination of the source of the differences between Eqs. (4) and (5) can be instructive. Figure 17 compares the

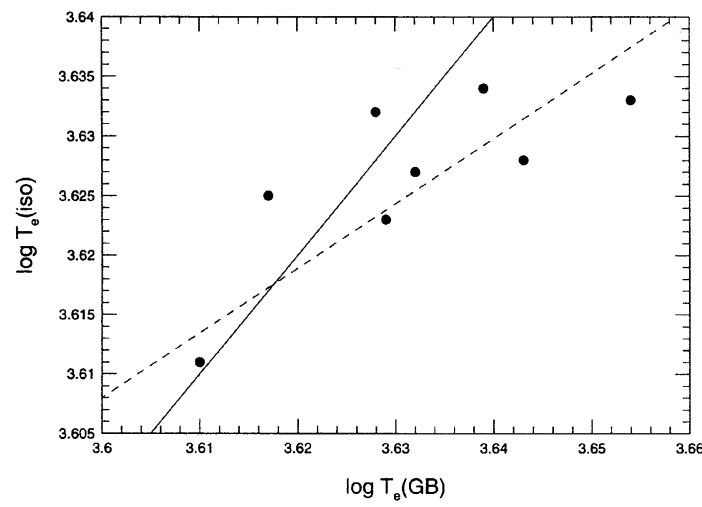

FIG. 17. Comparison of the empirical GB effective temperatures for the open clusters, $T_{c}$ (GB), and those predicted by the Revised Yale Isochrones, $T_{e}$ (iso), via Eq. (4) and the literature averages of $[\mathrm{Fe} / \mathrm{H}]$ and $\tau$ given in Table 1 . The dashed line shows the best linear fit between these two values of $T_{c}$; the solid line represents equality of the two temperatures. 
empirical $\log T_{e}$ (GB) values with those of $\log T_{e}$ (iso). A linear regression between these two temperatures has a correlation coefficient $r=0.77$ and is given by

$\log T_{e}^{\prime}$ (iso) $=1.646( \pm 0.192)$

$$
+0.545( \pm 0.134) \log T_{e}(\mathrm{~GB}) \text {. }
$$

This is the dashed line in Fig. 17; the solid line in the figure is the line of equality. NGC 2204 and NGC 2660 deviate from the line of equal temperatures in Fig. 17 by more than $\pm 0.010(\sim \pm 100 \mathrm{~K})$ in $\log T_{e}(\mathrm{~GB})$ and are responsible for nearly all of the skew in the observed $\log T_{e}(\mathrm{~GB})$, $\log T_{e}$ (iso) relationship. Note that neither of these clusters has had a proper motion study, and that NGC 2660 has the highest $\mathrm{E}(B-V)$ value in our sample. The smallest differences between the two $T_{e}$ values, on the other hand, are exhibited by the three clusters for which proper motion membership studies have been published; these clusters also have three of the four lowest reddenings of the eight open clusters observed.

In order to assess the likelihood that errors in $\mathrm{E}(B-V)$, $(m-M)_{0},[\mathrm{Fe} / \mathrm{H}]$ or $\tau$ could produce the differences in the empirical and theoretical GB temperatures of NGC 2204 and NGC 2660, we consult Eq. (4) and Table 12. NGC 2660 shows the largest difference between $\log T_{e}(\mathrm{~GB})$ and $\log T_{e}$ (iso), 0.021 (more than $200 \mathrm{~K}$ ), and its $\mathrm{GB}$ position is also the most uncertain of the clusters observed. The most reasonable means to produce agreement between the two temperatures for NGC 2660 is to reduce $\mathrm{E}(B-V)$ to 0.29 mag or $(m-M)_{0}$ to $11.6 \mathrm{mag}$; the age and metallicity adjustments required are too extreme to be plausible. Although no reddening determination published to date supports a value for $\mathrm{E}(B-V)$ as low as that required, the situation in NGC 2660 may be similar to that seen in NGC 2477; field star contamination of the cluster data may bias the reddening. However, the value used here places star 9016 on the field giant relation in Fig. 9(b), so a much lower reddening would be difficult to justify. Also, only two distance determinations for the cluster are less than $12.1 \mathrm{mag}$; one is Hartwick \& Hesser's (1971) fit of the normal points of NGC 2477 to a preliminary CMD for NGC 2660, and the other is based on Eggen's (1974) $M_{\text {bol }}$ determination for star 4304 only. Although 4304 is one of just two stars which we consider to lie on the GB in Fig. 8, it seems unlikely that the temperature discrepancy observed in NGC 2660 can be explained by uncertainties in the empirically determined parameters.

However, the disagreement between $\log T_{e}$ (GB) and $\log T_{e}$ (iso) in NGC 2660 could be quite naturally explained if the stars we have used to delineate the GB in the $H-R$ diagram are actually AGB stars. In other words, if the masses of the NGC 2660 giants are sufficiently large that the cluster has not yet experienced Renzini \& Buzzoni's (1986) RGB phase transition, as suggested by Barbaro \& Pigatto (1984) and as also proposed for NGC 2477 in Sec. 4.6, then the true GB position lies to the red of that shown in Fig. 14(d). If the separation between stars 2122 and 2212 and the chosen GB of NGC 2506 is representative of the AGB/GB temperature difference in open clusters, then the true value of $\log T_{e}$ (GB) for NGC 2660 may be approximately that predicted by the Revised Yale Isochrones. Mazzei \& Pigatto (1988) discuss the improved fit of the red giant luminosity function and the CMD of NGC 2660 by isochrones which include convective overshooting; the models used to construct these isochrones predict that the transition from quiet helium ignition to a helium flash at the RGB tip occurs at $\tau \sim 2.5 \mathrm{Gyr}$, much later than the age assumed for the NGC 2660 giants here. Of course, if the overshoot isochrones are truly more representative than the standard ones, agreement of the GB position with the prediction of the Revised Yale Isochrones is fortuitous but unnecessary.

If the offset of NGC 2204 from the line of equal temperatures in Fig. 17(a) is due to errors in its empirical parameters, the most likely candidates are too large a distance modulus or metallicity. The values these parameters must take to minimize the observed discrepancy are $(m-M)_{0}=12.6$ or $[\mathrm{Fe} / \mathrm{H}]=-0.70$. Of these two, a distance modulus error seems the most probable; the DDO work of Dawson (1981) and one fit of the CMD to a theoretical ZAMS (Barbaro \& Pigatto 1984) produce values for $(m-M)_{0}$ in the required range. Additionally, Fig. 13 shows that the clump stars of NGC 2204 lie $\sim 0.3 \mathrm{mag}$ brighter than those of the other clusters, possibly due to the use of an incorrect $(m-M)_{0}$. Meanwhile, the lowest metallicity estimate found for NGC 2204 is -0.62 dex by Cameron (1985). Thus, it could well be that uncertainties in $(m-M)_{0}$ and $[\mathrm{Fe} / \mathrm{H}]$ result in the displacement of NGC 2204 from the line of equality in Fig. 17(a). We note, though, that NGC 2204 is the third youngest open cluster we observed, and it may also lie on the high-mass side of the RGB phase transition. However, Barbaro \& Pigatto are able to self-consistently fit its red giant luminosity function and main-sequence turnoff parameters using standard evolutionary models.

If the inequality of the two temperature scales, $T_{e}$ (GB) and $T_{e}$ (iso), is real, it may be due to a mixing length to pressure scale height ratio $(\alpha)$ which decreases with increasing metallicity or mass (i.e., decreasing age); FPC81 discuss the possibility that $\alpha$ decreases with increasing metallicity. As seen previously, a variable $\alpha$ must make the isochrone temperature more dependent upon both $[\mathrm{Fe} / \mathrm{H}]$ and $\log \tau$ to produce agreement between Eqs. (4) and (5). Clearly, though, Fig. 17 shows that, at least for the range of age and metallicity covered by the open clusters in our study, there is little or no systematic error in the Revised Yale Isochrones (at $M_{\text {bol }}=-1.0$ ). The two temperatures agree for each of the eight clusters to within the uncertainty of \pm 0.023 estimated for $\log T_{e}$ (GB) in Sec. 6. Unfortunately, the small number of well observed, old open clusters again limits the conclusions we can draw from our study.

\section{SUMMARY}

We have presented new infrared photometry of giants in eight old open clusters. From these data and published optical data, we have determined effective temperatures, surface gravities, and bolometric luminosities for these stars and attempted to precisely locate the cluster giant branches in the $H-R$ diagram. The following conclusions result from this work:

(1) The infrared CMDs are similar in appearance to their optical counterparts but show less scatter along the GB. Although usually sparse, the infrared GBs can be fairly well defined because evidence suggests that many of the stars displaced from the GB are actually field interlopers.

(2) The giants from the most metal-poor open clusters overlap those from the most metal-rich globular clusters in metallicity and occupy a similar region in a $(U-V)_{0},(V-K)_{0}$ diagram; both groups of stars lie between the field giants and the giants from the metal-poor globular clusters. In addition, 
the stars in a particular cluster describe a fairly linear trend in this plot; we suggest that the $U V K$ plane may be useful as a membership discriminator for giants in moderately metalpoor, populous clusters with high quality $U V K$ photometry.

(3) The colors of giants in NGC 2420, NGC 2477, and NGC 2660 show systematic deviations from those of other giants in the $(J-K)_{0},(V-K)_{0}$ diagram; these deviations are probably caused by photometric errors, but we have been unable to identify the specific errors involved.

(4) The open cluster giants show considerable scatter in a $(\mathrm{J}-\mathrm{H})_{0},(\mathrm{H}-\mathrm{K})_{0}$ diagram but generally lie near the relation defined by field giants, rather than lying near that defined by globular cluster giants.

(5) The giants from a particular cluster define a fairly tight relationship between $\mathrm{CO}$ and $(V-K)_{0}$. In addition, the open cluster data strengthen the correlation between a cluster's metallicity and the average $\mathrm{CO}$ strength of its giants, which had previously been derived from globular cluster observations alone.

(6) The observed relation between age and metallicity for the open clusters in our sample is similar to that of LMC clusters. After adjusting the open cluster data for the galactic radial metallicity gradient, the open cluster age-metallicity relation lies between those of the LMC clusters and the solar neighborhood, perhaps indicating that the open cluster giants are members of the thick disk population.
(7) The open cluster GB temperatures at $M_{\text {bol }}=-1.0$ are generally in good agreement with the predictions of the Revised Yale Isochrones; uncertainties in the cluster parameters selected from the literature are most likely responsible for any discrepancies between the isochrones and the infrared GBs.

(8) The appearance of the $H-R$ diagram of NGC 2477 and the disagreement between the empirical and theoretical GB temperatures of NGC 2660 may indicate the presence of convective overshooting.

J. A. F. acknowledges the hospitality of Las Campanas Observatory, Carnegie Institution of Washington, while these observations were being made. We thank Eric Persson, who obtained some of these data and collaborated with us in the early phases of the project. We also express our gratitude to Dr. J.-C. Mermilliod for providing us with photometry of NGC 2477 stars (obtained in cooperation with Dr. J. J. Claria) and radial velocities of giants in NGC 2477 and NGC 2660 in advance of publication. We are indebted to Dr. R. D. Mathieu for allowing us to use his unpublished radial velocities of stars in NGC 2506. CTIO is operated by the Association of Universities for Research in Astronomy, Inc. under contract with the National Science Foundation.

\section{REFERENCES}

Anthony-Twarog, B. J. 1987, AJ, 93, 647

Anthony-Twarog, B. J., Kaluzny, J., Shara, M. M., \& Twarog, B. A. 1990, AJ, 99, 1504

Anthony-Twarog, B. J., Twarog, B. A., \& McClure, R. D. 1979, ApJ, 233, 188 (ATM)

Barbaro, G., \& Pigatto, L. 1984, A\&A, 136, 355

Bertelli, G., Bressan, A., \& Chiosi, C. 1985, A\&A, 150, 33

Bessell, M. S., Wood, P. R., \& Lloyd Evans, T. 1983, MNRAS, 202, 59 (BWLE)

Bonifazi, A., Fusi Pecci, F., Romeo, G., \& Tosi, M. 1990, MNRAS, 245, 15

Burstein, D., Faber, S. M., \& Gonzalez, J. J. 1986, AJ, 91, 1130

Cabrera-Caño, J., \& Alfaro, E. J. 1990, A\&A, 235, 94

Cameron, A. C., \& Reid, N. 1987, MNRAS, 224, 821

Cameron, L. M. 1985, A\&A, 147, 39

Cannon, R. D., \& Lloyd, C. 1970, MNRAS, 150, 279

Carlberg, R. G., Dawson, P. C., Hsu, T., \& VandenBerg, D. A. 1985, ApJ, 294, 674

Catchpole, R. M., \& Feast, M. W. 1973, MNRAS, 164, 11 P

Chiu, L.-T. G., \& van Altena, W. F. 1981, ApJ, 243, 827

Christian, C. A., Heasley, J. N., \& Janes, K. A. 1985, ApJ, 299, 683

Ciardullo, R. B., \& Demarque, P. 1977, Trans. Yale Univ. Obs., 33

Cohen, J. G. 1980, ApJ, 241, 981

Cohen, J. G. 1982, ApJ, 258, 143

Cohen, J. G., Frogel, J. A., \& Persson, S. E. 1978, ApJ, 222, 165 (CFP)

Cohen, J. G., Frogel, J. A., Persson, S. E., \& Elias, J. H. 1981, ApJ, 249, 481

Coleman, L. A. 1982, AJ, 87, 369

Dawson, D. W. 1978, AJ, 83, 1424

Dawson, D. W. 1981, AJ, 86, 237

Eggen, O. J. 1972a, ApJ, 172, 639

Eggen, O. J. 1972b, ApJ, 174, 45

Eggen, O. J. 1974, PASP, 86, 960

Eggen, O. J. 1983, AJ, 88, 813

Eggen, O. J., \& Sandage, A. R. 1964, ApJ, 140, 130 (ES)

Elias, J. H., Frogel, J. A., Hyland, A. R., \& Jones, T. J. 1983, AJ, 88, 1027

Fagerholm, E. 1906, inaugural dissertation, Uppsala

Foy, R., \& Proust, D. 1981, A\&A, 99, 221
Francic, S. P. 1989, AJ, 98, 888

Friel, E. D. 1989, PASP, 101, 244

Friel, E. D., Liu, T., \& Janes, K. A. 1989, PASP, 101, 1105

Frogel, J. A., Cohen, J. G., \& Persson, S. E. 1983, ApJ, 275, 773 (FCP)

Frogel, J. A., Mould, J., \& Blanco, V. M. 1990, ApJ, 352, 96

Frogel, J. A., Persson, S. E., Aaronson, M., \& Matthews, K. 1978, ApJ, 220, 75

Frogel, J. A., Persson, S. E., \& Cohen, J. G. 1981, ApJ, 246, 842 (FPC81)

Frogel, J. A., Persson, S. E., \& Cohen, J. G. 1983, ApJS, 53, 713 (FPC83)

Frogel, J. A., \& Twarog, B. A. 1983, ApJ, 274, 270

Geisler, D. 1987, AJ, 94, 84

Geisler, D. P., \& Smith, V. V. 1984, PASP, 96, 871

Girard, T. M., Grundy, W. M., Lopez, C. E., \& van Altena, W. F. 1989, AJ, 98, 227

Gratton, R. G., 1982, ApJ, 257, 640

Green, E. M., Demarque, P., \& King, C. R. 1987, The Revised Yale Isochrones and Luminosity Functions (Yale University Observatory, New Haven)

Hartwick, F. D. A., \& Hesser, J. E. 1971, PASP, 83, 53

Hartwick, F. D. A., \& Hesser, J. E. 1973, ApJ, 183, 883

Hartwick, F. D. A., \& Hesser, J. E. 1974, ApJ, 192, 391

Hartwick, F. D. A., Hesser, J. E., \& McClure, R. D. 1972, ApJ, 174, 557 (HHM)

Hawarden, T. G. 1975, MNRAS, 173, 801

Hawarden, T. G. 1976a, MNRAS, 174, 225

Hawarden, T. G. 1976b, MNRAS, 174, 471

Hawarden, T. G. 1978, MNRAS, 182, 31 P

Hesser, J. E., \& Smith, G. H. 1987, PASP, 99, 1044

Hirshfeld, A., McClure, R. D., \& Twarog, B. A. 1978, in The HR Diagram, IAU Symposium No. 80, edited by A. G. D. Philip \& D. S. Hayes (Reidel, Dordrecht), p. 163

Janes, K. A. 1974, ApJ, 189, 423

Janes, K. A. 1979, ApJS, 39, 135

Janes, K. A., \& Smith, G. H. 1984, AJ, 89, 487

Janes, K. A., Tilley, C., \& Lyngå, G. 1988, AJ, 95, 771

Johnson, H. L. 1966, ARA\&A, 4, 193 
Johnson, H. L., \& Sandage, A. R. 1955, ApJ, 121, 616

Joner, M. D., \& Taylor, B. J. 1988, AJ, 96, 218

Lee, T. A. 1970, ApJ, 162, 217

Levato, H., Malaroda, S., Garcia, B., Morrell, N., \& Solivella, G. 1990, ApJS, 72, 323

Liu, T., \& Janes, K. A. 1987, PASP, 99, 1076

Lyngå, G. 1987, Publ. Astron. Inst. Czech. Acad. Sci., 69, 121

Maeder, A., \& Meynet, G. 1989, A\&A, 210, 155

Mateo, M. 1988, in The Harlow-Shapley Symposium on Globular Cluster

Systems in Galaxies, IAU Symposium No. 126, edited by J. E. Grindlay

\& A. G. D. Philip (Kluwer, Dordrecht), p. 557

Mathieu, R. D., \& Latham, D. W. 1986, AJ, 92, 1364

Mathieu, R. D., \& Latham, D. W. 1990 (private communication)

Mathieu, R. D., Latham, D. W., Griffin, R. F., \& Gunn, J. E. 1986, AJ, 92, 1100

Mazzei, P., \& Pigatto, L. 1988, A\&A, 193, 148

McClure, R. D., Forrester, W. T., \& Gibson, J. 1974, ApJ, 189, 409

McClure, R. D., Newell, B., \& Barnes, J. V. 1978, PASP, 90, 170

McClure, R. D., Twarog, B. A., \& Forrester, W. T. 1981, ApJ, 243, 841

Mermilliod, J.-C. 1990 (private communication)

Mermilliod, J.-C., \& Claria, J. J. 1990 (private communication)

Mermilliod, J.-C., \& Mayor, M. 1989, A\&A, 219, 125

Mermilliod, J.-C., \& Mayor, M. 1990, A\&A, 237, 61

Murray, C. A., \& Clements, E. D. 1968, R. Obs. Bull. No. 139

Murray, C. A., Corben, P. M., \& Allchorn, M. R. 1965, R. Obs. Bull. No. 91

Nissen, P. E., Twarog, B. A., \& Crawford, D. L. 1987, AJ, 93, 634

Norris, J. E., \& Green, E. M. 1989, ApJ, 337, 272
Osborn, W. 1974, MNRAS, 168, 291

Pagel, B. E. J. 1974, MNRAS, 167, 413

Persson, S. E., Frogel, J. A., Cohen, J. G., Aaronson, M., \& Matthews, K. 1980, ApJ, 235, 452

Pilachowski, C. A., Saha, A., \& Hobbs, L. M. 1988, PASP, 100, 474

Racine, R. 1971, ApJ, 168, 393

Renzini, A., \& Buzzoni, A. 1986, in Spectral Evolution of Galaxies, edited by C. Chiosi and A. Renzini (Reidel, Dordrecht), p. 195

Sanders, W. L. 1977, A\&AS, 27, 89

Sarma, M. B. K., \& Walker, M. F. 1962, ApJ, 135, 11

Schild, R. 1983, PASP, 95, 1021

Schild, R. 1985, PASP, 97, 824

Smith, H. A., \& Hesser, J. E. 1983, PASP, 95, 277

Smith, V. V., \& Suntzeff, N. B. 1987, AJ, 93, 359

Sowell, J. R. 1987, ApJS, 64, 241

Sturch, C. 1972, PASP, 84, 666

Sturch, C. 1973, PASP, 85, 724

Sweigart, A. V., Greggio, L., \& Renzini, A. 1990, ApJ, 364, 527

Taylor, B. J., \& Joner, M. D. 1985, AJ, 90, 479

Taylor, B. J., \& Joner, M. D. 1988, AJ, 96, 211

Twarog, B. A. 1978, ApJ, 220, 890

Twarog, B. A., \& Anthony-Twarog, B. J. 1989, AJ, 97, 759

van Altena, W. F., \& Jones, B. F. 1970, A\&A, 8, 112

VandenBerg, D. A. 1985, ApJS, 58, 711

van den Bergh, S. 1977, ApJ, 215, 89

van den Bergh, S., \& McClure, R. D. 1980, A\&A, 88, 360

West, F. R. 1967, ApJS, 14, 384 